\title{
Ingoing Eddington-Finkelstein metric of an evaporating black hole
}

\author{
Shohreh Abdolrahimi, ${ }^{1,2, *}$ Don N. Page, ${ }^{1, \dagger}$ and Christos Tzounis ${ }^{1,2, *}$ \\ ${ }^{1}$ Theoretical Physics Institute, University of Alberta, Edmonton, Alberta T6G 2E1, Canada \\ ${ }^{2}$ Department of Physics and Astronomy, California State Polytechnic University, \\ 3801 West Temple Ave., Pomona, California 91768, USA
}

(Received 27 July 2017; published 16 December 2019)

\begin{abstract}
We present an approximate time-dependent metric in ingoing Eddington-Finkelstein coordinates for an evaporating nonrotating black hole as a first-order perturbation of the Schwarzschild metric, using the linearized backreaction from a realistic approximation for the stress-energy tensor for the Hawking radiation in the Unruh quantum state.

DOI: 10.1103/PhysRevD.100.124038
\end{abstract}

\section{INTRODUCTION}

The physics of black holes is an abundant field in which the convergence of gravitation, quantum theory, and thermodynamics takes place. The original derivation of Hawking radiation [1] from black holes is based on semiclassical effective field theory. Normally, quantum fields are considered test fields in the curved spacetime of a classical background geometry. A quantum field theory constructed on a curved background spacetime experiences gravitationally induced vacuum polarization and/or particle creation. These effects induce a nonzero expectation value for the stress-energy tensor. The renormalized expectation value of the complete quantum stressenergy tensor outside the classical event horizon has been calculated by various authors [2-43], usually using a framework established by Christensen and Fulling [3]. In this framework, the assumptions are that the stressenergy tensor is time independent, satisfies local stressenergy conservation, and has a trace determined solely by the conformal or Weyl anomaly [44] for fields that are classically conformally invariant (such as the conformally coupled massless scalar field and the electromagnetic field, but not the gravitational field [45]). The quantum state considered is usually either the Hartle-Hawking state [46] or the Unruh state [47,48]. (For discussions of the various quantum field theory states outside a black hole, see [49].) In the Hartle-Hawking state, one has thermal equilibrium,

\footnotetext{
*abdolrah@ualberta.ca

profdonpage@gmail.com

tzounis@ualberta.ca
}

Published by the American Physical Society under the terms of the Creative Commons Attribution 4.0 International license. Further distribution of this work must maintain attribution to the author(s) and the published article's title, journal citation, and DOI. Funded by SCOAP. and zero net energy flux, with the outgoing Hawking radiation balanced by incoming radiation from an external heat bath at the Hawking temperature. In [6], a fairly good closed-form approximation for the energy density and stresses of a conformal scalar field in the Hartle-Hawking state everywhere outside a static black hole can be found.

In the Unruh state, there is the absence of incoming radiation at both past null infinity and the past horizon, plus regularity of the stress-energy tensor on the future event horizon in the frame of a freely falling observer, representing a black hole formed from gravitational collapse, with nothing falling into the black hole thereafter. There have been many calculations of the quantum stress-energy tensor in the Unruh state in the Schwarzschild spacetime, both for a massless scalar field and for the electromagnetic field [8-12,17-23]. A method for computing the stress-energy tensor for the quantized massless spin- $1 / 2$ field in a general static spherically symmetric spacetime was presented in [24-26]. The quantum stress-energy tensor has also been investigated in the Kerr metric [22,28,36,38,42].

One of the important questions that one wants to answer concerns the effect of quantized matter on the geometry of black holes. Such effects in the Hartle-Hawking state have been studied [50] (for similar work, see [51-53]), using the approximation found in [6] for the expectation value of the renormalized thermal equilibrium stress-energy tensor of a free conformal scalar field in a Schwarzschild black hole background as the source in the semiclassical Einstein equation. The backreaction and new equilibrium metric are found perturbatively to first order in $\hbar$. The new metric is not asymptotically flat unless the system is enclosed by a reflecting wall. The nature of the modified black hole spacetime was explored in subsequent work [54-56]. James Bardeen [57] considered radial null geodesics in a black hole geometry modified by Hawking radiation backreaction, showing that the event horizon is stable and shifted slightly in radius from the vacuum background. 
In this paper, we construct the first-order backreaction on the metric in the Unruh state, using the expectation value of the quantum stress-energy tensor in the Unruh state as the source in the spherically symmetric Einstein equations. This metric represents the first-order approximation to the metric of an evaporating black hole.

\section{METRIC ANSATZ}

To construct a metric using the expectation value of the quantum stress-energy tensor in the Unruh state as a source in the spherically symmetric Einstein equations, we first need to find an appropriate metric ansatz. To do so, we begin (but do not end) with the outgoing Vaidya metric. The outgoing Vaidya metric describes a spherically symmetric spacetime with radially outgoing null radiation. Here, we consider a black hole evaporating by Hawking emission. The outgoing Vaidya metric can be written in outgoing Eddington-Finkelstein coordinates as

$$
d s^{2}=-\left(1-\frac{2 \mu(u)}{r}\right) d u^{2}-2 d u d r+r^{2} d \Omega^{2} .
$$

Here $u$ is the retarded or outgoing null time coordinate and $d \Omega^{2}=d \theta^{2}+\sin ^{2} \theta d \phi^{2}$. We use Planck units, $\hbar=c=$ $G=1$.

When the black hole mass $\mu$ is much larger than the reciprocal of the masses of all massive particles, the Hawking emission is almost entirely into massless particles (e.g., photons and gravitons for astrophysical mass black holes), and the Hawking emission rate is given by

$$
\mu^{\prime} \equiv \frac{d \mu}{d u} \approx-\frac{\alpha}{\mu^{2}},
$$

where $\alpha$ is a constant coefficient that has been numerically evaluated to be about $\alpha \approx 3.7474 \times 10^{-5}$ [58-62] for the emission of massless photons and gravitons. Then, we have

$$
\mu(u) \approx(-3 \alpha u)^{\frac{1}{3}} .
$$

We are setting $u=0$ at the final evaporation of the black hole, so that $u$ is negative for the part of the spacetime being considered. Moreover, we are assuming that the black hole mass is infinite at negative infinite $u$, so going back in retarded time, the mass grows indefinitely, rather than having a black hole that forms at some particular time. However, for a black hole that forms at some initial mass $M_{0}$, the metric we find should be good for values of $u$ when $\mu(u)<M_{0}$.

For $r \gg 2 \mu$, the retarded/outgoing time $u$ can be written in terms of the advanced/ingoing time $v$ and radius $r$ as approximately

$$
u \approx v-2(r-2 \mu)-4 \mu \ln \frac{r}{2 \mu} .
$$

Then $\mu(u)$ can be written in terms of $v$ and $r$ as well, since

$$
\begin{aligned}
\mu^{3} & \approx-3 \alpha v+6 \alpha r-12 \alpha \mu+12 \alpha \mu \ln \frac{r}{2 \mu} \\
& =-3 \alpha v+12 \alpha \mu\left(\frac{1}{z}-\ln z-1\right),
\end{aligned}
$$

where

$$
z \equiv \frac{2 \mu}{r}
$$

In terms of the zeroth-order solution

$$
\mu_{0}(v) \equiv(-3 \alpha v)^{\frac{1}{3}}
$$

and the function

$$
\epsilon(v, z) \equiv \frac{4 \alpha}{\mu_{0}^{2}}\left(\frac{1}{z}-\ln z-1\right)
$$

(which vanishes on the apparent horizon $r=2 \mu$ or $z=1$ ), the mass $\mu$ is given approximately by the solution of the cubic equation (5) as

$\mu(v, z) \approx \mu_{0}\left[\frac{1}{2}+\left(\frac{1}{4}-\epsilon^{3}\right)^{\frac{1}{2}}\right]^{\frac{1}{3}}+\mu_{0}\left[\frac{1}{2}-\left(\frac{1}{4}-\epsilon^{3}\right)^{\frac{1}{2}}\right]^{\frac{1}{3}}$.

Figure 1 shows a spacetime diagram for the evaporating black hole and the null coordinates $u$ and $v$. These null coordinates are chosen so that $u=0$ is the event horizon of

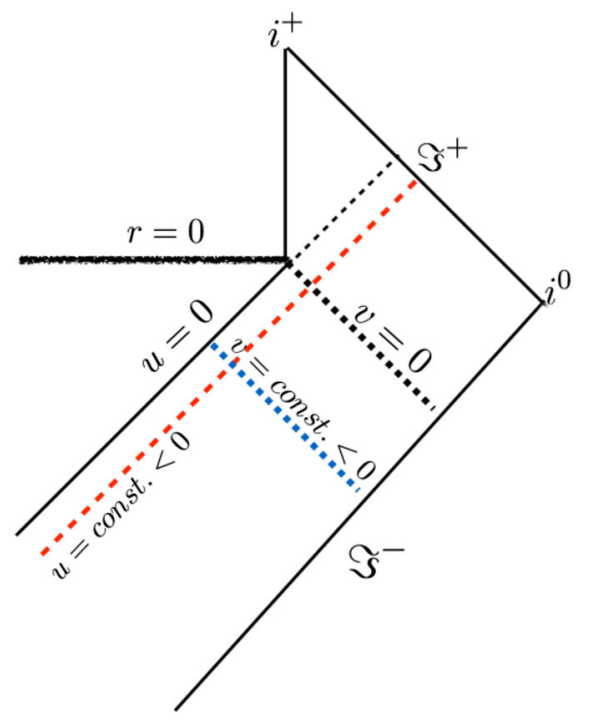

FIG. 1. Carter-Penrose diagram for an evaporating black hole. The black hole mass is infinite at negative infinite $u$. Our metric applies for $v \ll-1$ (in Planck units) and for $u<0$, though it should also be good for $u$ somewhat positive (a bit inside the black hole, so long as $z \equiv 2 \mu / r$ is not much larger than unity). 
the black hole, and $u=v=0$ is the final evaporation event of the hole, when and where the horizon radius goes to $r=0$. We shall consider the region outside the black hole horizon, where $r>2 \mu$, so $0<z<1$, though our results should continue to be approximately valid somewhat inside the black hole, so long as $z \equiv 2 \mu / r$ is not too much larger than unity, since the approximations used later for the stress-energy tensor were derived for $z<1$ and are expected to become poor for $z$ significantly larger than unity, though there is no singularity or discontinuity at the horizon itself.

When $\epsilon \ll 1$, Eq. (9) implies that

$\mu(v, z) \approx \mu_{0}(1+\epsilon)=(-3 \alpha v)^{\frac{1}{3}}+\frac{4 \alpha}{(-3 \alpha v)^{\frac{1}{3}}}\left(\frac{1}{z}-\ln z-1\right)$.

If $-3 \alpha v \gg 1$, then Eq. (7) implies that $\mu_{0} \gg 1$ (here 1 being the Planck mass in the Planck units we are using), the condition that the semiclassical approximation be good everywhere outside (and also somewhat inside) the black hole. In this case, Eq. (8) implies that $\epsilon \ll 1$ unless $z \lesssim 4 \alpha / \mu_{0}^{2} \ll 1$, in which case $1 / z \gg-\ln z$, so that $\epsilon \approx 4 \alpha /$ $\left(\mu_{0}^{2} z\right)=2 \alpha r /\left(\mu_{0}^{2} \mu\right)<2 \alpha r /\left(\mu_{0}^{3}\right)=2 r /(-3 v)$. Therefore, if $r \ll-3 v / 2 \gg 1 / \alpha$, then $\epsilon \ll 1$. This condition is implied by $0<-u \ll-4 v \gg 4 /(3 \alpha)$, since then Eq. (4) gives $2 r \approx v-u-4 \mu \ln [r /(2 \mu)]<-u-(-v) \ll 3(-v)$. That is, $0<-u \ll-4 v \gg 4 /(3 \alpha)$ is sufficient to imply $\epsilon \ll 1$ and the validity of the approximation of Eq. (10). (The approximation also continues to be good slightly inside the horizon, where $u>0$.)

With $-3 \alpha v \gg 1$, the second term on the right-hand side of Eq. (10) is insignificant unless $z \lesssim 4 \alpha / \mu_{0}^{2} \ll 1$, and for
$4 \alpha(-3 \alpha v)^{-\frac{2}{3}} \ll z \ll 1$, we can take $z \approx 2 \mu_{0} / r$, so that in any case in which $-3 \alpha v \gg 1$ and $z \gg 4 \alpha(-3 \alpha v)^{-\frac{2}{3}}$,

$$
\begin{aligned}
\mu(v, r) \approx & \mu_{0}+\frac{2 \alpha\left(r-2 \mu_{0}\right)}{\mu_{0}^{2}}+\frac{4 \alpha}{\mu_{0}} \ln \frac{r}{2 \mu_{0}} \\
\equiv & (-3 \alpha v)^{\frac{1}{3}}+2 \alpha r(-3 \alpha v)^{-\frac{2}{3}}-4 \alpha(-3 \alpha v)^{-\frac{1}{3}} \\
& +4 \alpha(-3 \alpha v)^{-\frac{1}{3}} \ln \frac{r}{2(-3 \alpha v)^{\frac{1}{3}}} .
\end{aligned}
$$

Next, we transform the outgoing Vaidya metric to ingoing Eddington-Finkelstein coordinates with advanced/ ingoing time $v$ and radius $r$. Let us consider $u$ as a function of $v$ and $r$ again as in Eq. (4). Then, we have

$$
\begin{aligned}
d u & \approx d v-2 d r-4 \mu^{\prime} \ln \frac{r}{2 \mu} d u-\frac{4 \mu d r}{r}+8 \mu^{\prime} d u \\
& \Rightarrow d u \approx e^{\tilde{\psi}(v, r)} d v-2\left(1+\frac{2 \mu}{r}\right) e^{\tilde{\psi}(v, r)} d r
\end{aligned}
$$

where

$$
e^{-\widetilde{\psi}(v, r)} \equiv 1-8 \mu^{\prime}+4 \mu^{\prime} \ln \frac{r}{2 \mu}
$$

For $0<-u \ll-4 v \gg 4 /(3 \alpha)$ so that $\epsilon \ll 1$ and hence $\mu-\mu_{0} \ll \mu_{0}$,

$$
\tilde{\psi}(v, r) \approx-4 \mu^{\prime}\left(\ln \frac{r}{2 \mu}-2\right) \approx-\frac{4 \alpha}{\mu^{2}}(\ln z+2) .
$$

Plugging back Eq. (12) into the metric (1), we get

$$
\begin{aligned}
d s^{2} \approx & -\left(1-\frac{2 \mu}{r}\right) e^{2 \tilde{\psi}(v, r)} d v^{2}+2 e^{\tilde{\psi}(v, r)}\left[2\left(1+\frac{2 \mu}{r}\right)\left(1-\frac{2 \mu}{r}\right) e^{\tilde{\psi}(v, r)}-1\right] d v d r \\
& +4\left(1+\frac{2 \mu}{r}\right) e^{\tilde{\psi}(v, r)}\left[1-\left(1+\frac{2 \mu}{r}\right)\left(1-\frac{2 \mu}{r}\right) e^{\tilde{\psi}(v, r)}\right] d r^{2}+r^{2} d \Omega^{2}
\end{aligned}
$$

For $1 \ll 2 \mu_{0} \ll r \ll-3 v / 2$, we thus have

$d s^{2} \approx-\left(1-\frac{2 \mu}{r}\right) e^{2 \tilde{\psi}(v, r)} d v^{2}+2 e^{\tilde{\psi}(v, r)} d r d v+r^{2} d \Omega^{2}$.

When $2 \mu_{0} \ll r$ is not true, one is close enough to the black hole that the small deviations of the metric from the Schwarzschild metric are not well approximated by the Vaidya metric, since the stress-energy tensor is not well approximated by purely outgoing null radiation as it would be in the Vaidya metric. However, for $1 \ll 2 \mu_{0}$, the metric is close to a slowly varying Schwarzschild metric, so it is an excellent approximation to solve the
Einstein equation as a deviation from Schwarzschild to linear order in the stress-energy tensor of the Hawking radiation, which itself can be well approximated by what it would be for a fixed Schwarzschild metric background. Furthermore, when one goes out from the black hole along the past-directed null direction with $v=$ const $\ll-1$, the energy of the Hawking radiation that one crosses increases the mass so that it is no longer well approximated by $\mu_{0}(v) \equiv(-3 \alpha v)^{\frac{1}{3}}$, as $z \equiv 2 \mu / r$ becomes sufficiently small that $\epsilon(v, z) \equiv\left(4 \alpha / \mu_{0}^{2}\right)\left(z^{-1}-\right.$ $\ln z-1)$ does not remain small. Then Eq. (14) is no longer a good approximation for what $\tilde{\psi}(v, r)$ should be. 
Therefore, we modify the metric (16) with corrections going as $1 / \mu^{2}$ with coefficients going mainly as functions of $z$ in order to match the stress-energy tensor of the Hawking radiation under the approximation that it is produced by a slowly varying sequence of Schwarzschild metrics. In particular, we write the general spherically symmetric metric in ingoing Eddington-Finkelstein coordinates as

$$
\begin{aligned}
d s^{2}= & -e^{2 \psi(v, z)}\left(1-\frac{2 m(v, z)}{r}\right) d v^{2}+2 e^{\psi(v, z)} d v d r \\
& +r^{2} d \Omega^{2}
\end{aligned}
$$

and motivated by the approximate forms above for the variables $\mu$ and $\tilde{\psi}$ in the metric (16), we make the following ansatz for the metric coefficients of the evaporating black hole:

$$
\begin{gathered}
\psi \approx \frac{1}{\mu_{0}^{2}}[g(z)-4 \alpha \ln \tilde{z}], \\
m \approx \mu\left[1+\frac{h(z)}{\mu_{0}^{2}}\right],
\end{gathered}
$$

where $\mu(v, z)$ is given by Eq. (9), or approximately by Eq. (10) for $2 r \ll-3 v, z \equiv 2 \mu / r$, and $z$ is a function of $\mu / \mu_{0}$ that is approximately $z$ for $2 r \ll-3 v$ but whose form for larger $r$ will be evaluated later.

Once we find the functions $g(z), h(z)$, and $\tilde{z}\left(\mu / \mu_{0}\right)$ to solve the Einstein equation for the small deviation of the metric from a sequence of Schwarzschild metrics with a slowly decreasing mass, the resulting metric (17) should be a good approximation for the exact nonrotating black hole metric for all $v \ll-1$ (i.e., for all advanced times before the black hole shrinks down to near the Planck mass) and for $z \equiv 2 \mu / r \lesssim 1$ (i.e., for all radii outside and even slightly inside the black hole), since the mass will be changing at a timescale much longer than the timescale of the mass itself. For $r \gg 1$ (radii large in Planck units), the metric should also be good for $v \gtrsim-1$ [at advanced times after the black hole shrinks down to near the Planck mass, where the metric has the approximate outgoing Vaidya form of Eq. (1)]. That is, the metric (17) with $\psi$ given by Eq. (18) and $m$ given by Eq. (19), with $g(z), h(z)$, and $\tilde{z}\left(\mu / \mu_{0}\right)$ to be found below, should be a good approximation for the metric everywhere outside, and even slightly inside, a black hole evaporating from infinite mass, except in the spacetime region near the final evaporation event of the black hole and to the causal future of this region (where one would expect quantum gravity effects to be important).

In summary, we started with the outgoing Vaidya metric as a first approximation for a spherically symmetric black hole metric evaporating by the emission of massless Hawking radiation, and then we switched to ingoing EddingtonFinkelstein coordinates and introduced the functions $g(z)$ and $h(z)$ to allow us to get a better approximation for the metric of an evaporating black hole with the stress-energy tensor of massless Hawking radiation, valid everywhere outside the black hole (and also slightly inside) that is not to the causal future of the Planckian region where the black hole has shrunk to near the Planck mass.

For $z=1$, we shall make the gauge choice of setting $g(1)=h(1)=\ln \tilde{z}(1)=0$, so that there $2 m \approx 2 \mu=r$ and $\psi \approx 0 . z=1$ is then the approximate location of the apparent horizon. For $z \rightarrow 0$ (radial infinity backwards along the ingoing radial null curve of fixed $v$ ), we have $g(z), h(z)$, and $\tilde{z}$ approaching the constants $g_{0}, h_{0}$, and $\tilde{z}_{0}$, respectively, so in this limit of infinitely large $r / \mu, m / \mu \rightarrow$ $1+h_{0} / \mu_{0}^{2}$ and $\psi \rightarrow\left(g_{0}-4 \alpha \ln \tilde{z}_{0}\right) / \mu_{0}^{2}$. It is only an approximation that $\psi$ and $m$ are functions just of $\mu$ and $z$ of this form, but for a large and hence very slowly evaporating black hole, it seems to be a very good approximation.

For $r \ll-v=\mu_{0}^{3} /(3 \alpha)$, which implies that $\mu \approx \mu_{0} \equiv$ $(-3 \alpha v)^{\frac{1}{3}}$, Eqs. (2) and (19) above show that at fixed $z$ the mass of the black hole changes as

$$
\frac{d m}{d v} \approx-\frac{\alpha}{\mu^{2}} .
$$

From Eqs. (6)-(9) and (17)-(19), we get that the area of the apparent horizon (at $z=1$ ) of the evaporating black hole is

$$
\begin{aligned}
A(v) & =4 \pi[2 m(v, r=2 m)]^{2} \approx 4 \pi[2 \mu(v, z=1)]^{2} \\
& =16 \pi \mu_{0}^{2}=16 \pi(-3 \alpha v)^{\frac{2}{3}} .
\end{aligned}
$$

Note that Bardeen [57] considers a quasistationary approximation of the black hole, which is justified as long as the black hole mass is much larger than the Planck mass $m_{p} \equiv(\hbar c / G)^{1 / 2}$, which we are setting equal to unity by using Planck units. In this case, Bardeen has the black hole mass at $r \sim 2 \mu_{0}, M \equiv \mu\left(v, r=2 \mu_{0}\right)$, being proportional to $(-v)^{1 / 3}$.

The metric (17) can be written in the form

$$
d s^{2}=-e^{2 \Psi}\left(1-\frac{2 \tilde{M}}{r}\right) d v^{2}+2 e^{\Psi} d v d r+r^{2} d \Omega^{2},
$$

where $\Psi \equiv \Psi(v, r)=\psi(v, z=2 \mu / r)$ and $\tilde{M} \equiv \tilde{M}(v, r)=$ $m(v, z=2 \mu / r)$. Components of the Einstein tensor for this metric have the following form:

$$
\begin{gathered}
G_{v}^{v}=-\frac{2}{r^{2}} \tilde{M}_{, r}, \\
G_{v}^{r}=\frac{2}{r^{2}} \tilde{M}_{, v}, \\
G_{r}^{v}=\frac{2}{r} e^{-\Psi} \Psi_{, r},
\end{gathered}
$$




$$
\begin{gathered}
G_{r}^{r}=\left(1-\frac{2 \tilde{M}}{r}\right) \frac{2 \Psi_{, r}}{r}-\frac{2 \tilde{M}_{, r}}{r^{2}}, \\
G_{\phi}^{\phi}=G_{\theta}^{\theta}=\left(1-\frac{2 \tilde{M}}{r}\right)\left(\Psi_{, r}^{2}+\Psi_{, r r}\right) \\
+\frac{1}{r}\left(1+\frac{\tilde{M}}{r}-3 \tilde{M}_{, r}\right) \Psi_{, r}-\frac{1}{r} \tilde{M}_{, r r}+e^{-\Psi_{, r v}} .
\end{gathered}
$$

Taking $v$ and $z$ to be the independent coordinates (at fixed angles $\theta$ and $\phi$ ), we can rewrite the metric (17) for $2 r \ll-3 v$ [the region where $g(z), h(z)$, and $z$ have significant variation; outside this region we can set $g \approx g_{0}, h \approx h_{0}$, and $\tilde{z} \approx \tilde{z}_{0}$ in the metric (17)] as

$$
\begin{aligned}
d s^{2} \approx & -\left[e^{2 \psi(v, z)}\left(1-z\left(1+\frac{h(z)}{\mu_{0}^{2}}\right)\right)-2 e^{\psi(v, z)} r_{, v}\right] d v^{2} \\
& +2 e^{\psi(v, z)} r_{, z} d z d v+\frac{4 \mu^{2}}{z^{2}} d \Omega^{2} \\
= & -A d v^{2}+2 B d v d z+r^{2} d \Omega^{2}
\end{aligned}
$$

where

$$
\begin{gathered}
r_{, v} \approx-\frac{2 \alpha}{z(-3 \alpha v)^{\frac{2}{3}}}+\frac{8 \alpha^{2}}{z^{2}} \frac{1-z \ln z-z}{(-3 \alpha v)^{\frac{4}{3}}}, \\
r_{, z} \approx-\frac{2}{z^{2}}(-3 \alpha v)^{\frac{1}{3}}-\frac{8 \alpha 2-z \ln z}{z^{3}} \frac{2-3 \alpha v)^{\frac{1}{3}}}{(-3}
\end{gathered}
$$

Although this form of the metric does not apply everywhere outside a large black hole as the metric (17) does, it applies where the stress-energy tensor of the Hawking radiation gives significant contributions to the functions $g(z), h(z)$, and $z$ in Eqs. (18) and (19). Therefore, this form of the metric will be used to get approximate solutions of the Einstein equations for $g(z), h(z)$, and $\tilde{z}$ with the stressenergy tensor of the Hawking radiation of massless fields in the Unruh quantum state. Then these will be inserted back into Eqs. (18) and (19) to give the functions in the metric (17), which will be a good approximation for the metric everywhere outside a large black hole evaporating by Hawking radiation of massless fields.

The stress-energy tensor for the spherically symmetric Unruh quantum state $|\psi\rangle$ on the spherically symmetric curved background of the Schwarzschild spacetime with $2 \mu \equiv r z$ constant,

$$
d s^{2}=-(1-z) d t^{2}+\frac{d r^{2}}{1-z}+r^{2} d \Omega^{2},
$$

in the standard static orthonormal frame

$$
\begin{gathered}
\omega^{\hat{0}}=\sqrt{1-z} d t, \\
\omega^{\hat{1}}=\frac{1}{\sqrt{1-z}} d r, \\
\omega^{\hat{2}}=r d \theta, \quad \omega^{\hat{3}}=r \sin \theta d \phi,
\end{gathered}
$$

can be written in the following form:

$$
\left\langle\psi\left|T^{\hat{\mu} \hat{\nu}}\right| \psi\right\rangle=\left(\begin{array}{cccc}
\tilde{\rho} & \tilde{f} & 0 & 0 \\
\tilde{f} & \tilde{P} & 0 & 0 \\
0 & 0 & \tilde{p} & 0 \\
0 & 0 & 0 & \tilde{p}
\end{array}\right)
$$

Note that we are using a capital $\tilde{P}$ for the radial pressure and a lowercase $\tilde{p}$ for the transverse pressure. Dimensional analysis shows that for massless fields at fixed $z$, the dependence of the orthonormal components of the stressenergy tensor on the mass $\mu$ of the Schwarzschild metric at fixed $z$ goes as $\mu^{-4}$, so for the slowly evolving metric (28), we shall assume that the stress-energy tensor (35) has approximately the following form with functions $\rho(z)$, $f(z), P(z)$, and $p(z)$ that are dimensionless even without setting $G=1$ (and hence independent of the scale set by $\mu_{0}$ ):

$$
\left\langle\psi\left|T^{\hat{\mu} \hat{\nu}}\right| \psi\right\rangle=\frac{1}{\mu_{0}^{4}}\left(\begin{array}{cccc}
\rho(z) & f(z) & 0 & 0 \\
f(z) & P(z) & 0 & 0 \\
0 & 0 & p(z) & 0 \\
0 & 0 & 0 & p(z)
\end{array}\right)
$$

According to Christensen and Fulling [3], the stressenergy tensor in the case of the Schwarzschild spacetime can be decomposed into four separately conserved quantities. We follow the analysis of Matt Visser [21] and use his form of the stress-energy tensor, which has a slightly different basis for its decomposition from that of Christensen and Fulling and is given by

$\left\langle\psi\left|T^{\hat{\mu} \hat{\nu}}\right| \psi\right\rangle=\left[T_{\text {trace }}\right]^{\hat{\mu} \hat{\nu}}+\left[T_{\text {pressure }}\right]^{\mu^{\hat{\nu}}}+\left[T_{+}\right]^{\hat{\mu} \hat{\nu}}+\left[T_{-}\right]^{\hat{\mu} \hat{\nu}}$,

where, with $T(z), H(z)$, and $G(z)$ being a further set of functions of $z \equiv 2 \mu / r$ that are dimensionless without setting the Newtonian gravitational constant to be unity, and with $f_{+}$and $f_{-}$being dimensionless constants, 


$$
\begin{gathered}
\mu_{0}^{4}\left[T_{\text {trace }}\right]^{\hat{\mu} \hat{\nu} \equiv}\left(\begin{array}{cccc}
-T(z)+\frac{z^{2}}{1-z} H(z) & 0 & 0 & 0 \\
0 & \frac{z^{2}}{1-z} H(z) & 0 & 0 \\
0 & 0 & 0 & 0 \\
0 & 0 & 0 & 0
\end{array}\right), \\
H(z) \equiv \frac{1}{2} \int_{z}^{1} \frac{T(\bar{z})}{\bar{z}^{2}} d \bar{z}
\end{gathered}
$$

$$
\begin{gathered}
\mu_{0}^{4}\left[T_{\text {pressure }}\right]^{\hat{\mu} \hat{\nu}} \\
\equiv\left(\begin{array}{cccc}
2 p(z)+\frac{z^{2}}{1-z} G(z) & 0 & 0 & 0 \\
0 & \frac{z^{2}}{1-z} G(z) & 0 & 0 \\
0 & 0 & p(z) & 0 \\
0 & 0 & 0 & p(z)
\end{array}\right), \\
G(z) \equiv \int_{z}^{1}\left[\frac{2}{\bar{z}^{3}}-\frac{3}{\bar{z}^{2}}\right] p(\bar{z}) d \bar{z} \\
\mu_{0}^{4}\left[T_{+}\right]^{\hat{\mu} \hat{\nu}} \equiv f_{+} \frac{z^{2}}{1-z}\left(\begin{array}{cccc}
1 & 1 & 0 & 0 \\
1 & 1 & 0 & 0 \\
0 & 0 & 0 & 0 \\
0 & 0 & 0 & 0
\end{array}\right), \\
\mu_{0}^{4}\left[T_{-}\right]^{\hat{\mu} \hat{\nu}} \equiv f_{-} \frac{z^{2}}{1-z}\left(\begin{array}{cccc}
1 & -1 & 0 & 0 \\
-1 & 1 & 0 & 0 \\
0 & 0 & 0 & 0 \\
0 & 0 & 0 & 0
\end{array}\right) .
\end{gathered}
$$

The decompositions (38) and (40) make sense if the integrals $G(z)$ and $H(z)$ converge. Imposing mild integrability constraints on $T(z)$ and $p(z)$ at the apparent horizon that is very near $z=1$, which are satisfied for the Unruh state where $T(1)$ and $p(1)$ are actually finite, we have

$$
\begin{aligned}
& H(z)=\frac{1}{2} T(1)(1-z)+O\left[(1-z)^{2}\right], \\
& G(z)=-p(1)(1-z)+O\left[(1-z)^{2}\right] .
\end{aligned}
$$

This is enough to imply that the two tensors (38) and (40) are individually regular at both the past and future horizon. In Kruskal null coordinates, $\left[T_{+}\right]$is singular on the future horizon $\mathrm{H}^{+}$and regular on the past horizon $\mathrm{H}^{-}$. On the other hand, $\left[T_{-}\right]$is singular on the past horizon $H^{-}$and regular on the future horizon $\mathrm{H}^{+}$. The two tensors (42) and (43) correspond to outgoing and ingoing null fluxes, respectively. The constants $f_{+}$and $f_{-}$determine the overall flux. The Unruh state must be regular on the future horizon, so we need $f_{+}=0$. However, such a condition naïvely seems to exclude any outgoing radiation. Nevertheless, we can get outgoing radiation by making $f_{-}$negative. It is convenient to define $\beta$ to be what $p(0)=\mu^{4} \tilde{p}(r=\infty)$ would be for massless scalar radiation in the HartleHawking state at large radii (ignoring the backreaction to the Schwarzschild metric),

$$
\beta \equiv \frac{1}{2^{13} 3^{2} 5 \pi^{2}} \equiv \frac{1}{368640 \pi^{2}},
$$

and set $f_{-}=-\beta f_{0}$, where $f_{0}$ is a positive quantity. In what follows, we also define

$$
f(z) \equiv \beta f_{0} \frac{z^{2}}{1-z} .
$$

Thus,

$\mu_{0}^{4}\left[T_{+}\right]^{\hat{\mu} \hat{\nu}}+\mu_{0}^{4}\left[T_{-}\right]^{\hat{\mu} \hat{\nu}} \equiv\left(\begin{array}{cccc}-f(z) & f(z) & 0 & 0 \\ f(z) & -f(z) & 0 & 0 \\ 0 & 0 & 0 & 0 \\ 0 & 0 & 0 & 0\end{array}\right)$.

Therefore, we have

$$
\begin{gathered}
\mu_{0}^{4} T^{\hat{0} \hat{0}}=\rho(z)=2 p(z)+\frac{z^{2}}{1-z}[H(z)+G(z)] \\
-T(z)-f(z), \\
\mu_{0}^{4} T^{\hat{1} \hat{0}}=\mu_{0}^{4} T^{\hat{0} \hat{1}}=f(z), \\
\mu_{0}^{4} T^{\hat{1} \hat{1}}=P(z)=\frac{z^{2}}{1-z}[H(z)+G(z)]-f(z), \\
\mu_{0}^{4} T^{\hat{2} \hat{2}}=\mu_{0}^{4} T^{\hat{3} \hat{3}}=p(z) .
\end{gathered}
$$

Since the stress-energy tensor is given in the orthonormal frame, we rewrite the Einstein equation in the orthonormal frame, i.e.,

$$
G_{\hat{\mu} \hat{\nu}}=8 \pi T_{\hat{\mu} \hat{\nu}}
$$

To find the approximate time-dependent metric for an evaporating black hole as a first-order perturbation of the Schwarzschild metric, using the linearized backreaction from the stress-energy tensor (49)-(52) of the Hawking radiation in the Unruh quantum state in the Schwarzschild spacetime, we solve Eq. (53) up to relative corrections of the order of $1 / \mu^{2}$ in the Planck units that we are using. To bring $G_{\mu \nu}$ to the orthonormal frame, note that we have

$$
\begin{aligned}
d s^{2} & =-\left(\omega^{\hat{0}}\right)^{2}+\left(\omega^{\hat{1}}\right)^{2}+\left(\omega^{\hat{2}}\right)^{2}+\left(\omega^{\hat{3}}\right)^{2} \\
& =-A d v^{2}+2 B d v d z+r^{2} d \Omega^{2},
\end{aligned}
$$


where

$$
\begin{gathered}
\omega^{\hat{0}}=\sqrt{A} d v-\frac{B}{\sqrt{A}} d z, \\
\omega^{\hat{1}}=\frac{B}{\sqrt{A}} d z, \\
\omega^{\hat{2}}=r d \theta, \quad \omega^{\hat{3}}=r \sin \theta d \phi .
\end{gathered}
$$

We have that the Einstein tensor is

$$
\begin{aligned}
G= & G_{\hat{0} \hat{0}} \omega^{\hat{0}} \omega^{\hat{0}}+G_{\hat{0} \hat{1}} \omega^{\hat{0}} \omega^{\hat{1}}+G_{\hat{1} \hat{0}} \omega^{\hat{1}} \omega^{\hat{0}}+G_{\hat{1} \hat{1}} \omega^{\hat{1}} \omega^{\hat{1}} \\
& +G_{\hat{2} \hat{2}} \omega^{\hat{2}} \omega^{\hat{2}}+G_{\hat{3} \hat{3}} \omega^{\hat{3}} \omega^{\hat{3}} \\
= & G_{v v} d v^{2}+2 G_{v z} d v d z+G_{z z} d z^{2}+G_{\theta \theta} d \theta^{2}+G_{\phi \phi} d \phi^{2} .
\end{aligned}
$$

Therefore, we obtain

$$
\begin{gathered}
G_{\hat{0} \hat{0}}=\frac{G_{v v}}{A}, \\
G_{\hat{0} \hat{1}}=G_{\hat{1} \hat{0}}=\frac{G_{v z}}{B}+\frac{G_{v v}}{A}, \\
G_{\hat{1} \hat{1}}=\frac{A G_{z z}}{B^{2}}+2 \frac{G_{v z}}{B}+\frac{G_{v v}}{A}, \\
G_{\hat{\imath} \hat{2}}=\frac{G_{\theta \theta}}{r^{2}}, \\
G_{\hat{\hat{\jmath}} \hat{\jmath}}=\frac{G_{\phi \phi}}{r^{2} \sin ^{2} \theta} .
\end{gathered}
$$

Deriving the Einstein tensor components $G_{v v}, G_{v z}, G_{z z}$, and $G_{\theta \theta}$ for the metric (28) and using Eqs. (59)-(63), we obtain the Einstein tensor components in the orthonormal frame.

\section{SOLUTION FOR THE METRIC COEFFICIENTS}

We now solve the Einstein equation (53) to first order in the perturbation of the metric from the Schwarzschild metric, using the stress-energy tensor whose components are proportional to $1 / \mu^{4}$. From the zero-one component of the Einstein equation, we get

$$
f(z)=\frac{\alpha z^{2}}{16 \pi(1-z)} .
$$

Comparing this to Eq. (47), we have $f_{0}=\alpha /(16 \pi \beta)$. Therefore, the Hawking radiation luminosity of the black hole is not only $L=-d m / d v \approx \alpha / \mu^{2}$ but also $16 \pi \beta f_{0} / \mu^{2}$. From the zero-zero component, we find

$$
\begin{aligned}
\rho(z) & =\frac{z^{2}}{32 \pi(1-z)}\left[2 \alpha\left(1-2 z^{2}\right)-z^{2}(1-z) h_{, z}\right] \\
& =f(z)\left(1-2 z^{2}\right)-\frac{z^{4} h_{, z}}{32 \pi} .
\end{aligned}
$$

From the one-one component of the Einstein equation (53), we get

$$
\begin{aligned}
P(z)= & \frac{z^{2}}{32 \pi(1-z)} \\
& \times\left[2 \alpha\left(1-8 z+6 z^{2}\right)-2 z(1-z)^{2} g_{, z}+z^{2}(1-z) h_{, z}\right] \\
= & f(z)\left(1-8 z+6 z^{2}\right)-\frac{1}{16 \pi} z^{3}(1-z) g_{, z}+\frac{1}{32 \pi} z^{4} h_{, z} .
\end{aligned}
$$

From the two-two component, we get

$$
\begin{aligned}
p(z)= & \frac{z^{3}}{64 \pi}\left[16 \alpha+(2-5 z) g_{, z}-2 z h_{, z}\right. \\
& \left.+2 z(1-z) g_{, z z}-z^{2} h_{, z z}\right] \\
= & 4 f(z) z(1-z) \\
& +\frac{z^{3}}{64 \pi}\left[(2-5 z) g_{, z}-2 z h_{, z}+2 z(1-z) g_{, z z}-z^{2} h_{, z z}\right] .
\end{aligned}
$$

Now let us suppose that in the stress-energy tensor components (49)-(52), the functions $p(z)$ and $T(z)$ are explicitly given. We then solve for the metric functions $h(z)$ and $g(z)$ in terms of $p(z)$ and $T(z)$. Moreover, we are making the gauge choice of setting $g(1)=h(1)=0$. From Eq. (64), we have $f(z)$.

From $G_{\hat{0} \hat{o}}=8 \pi T_{\hat{0} \hat{o}}$ and $G_{\hat{1} \hat{1}}=8 \pi T_{\hat{1} \hat{1}}$, we get

$$
\begin{aligned}
& g(z)=- \int_{z}^{1}\left[-\frac{2 \alpha\left(1-4 \bar{z}+2 \bar{z}^{2}\right)}{\bar{z}(1-\bar{z})^{2}}+16 \pi \frac{P(\bar{z})+\rho(\bar{z})}{\bar{z}^{3}(1-\bar{z})}\right] d \bar{z} \\
&=-\int_{z}^{1}\left[32 \pi \frac{H(\bar{z})+G(\bar{z})}{\bar{z}(1-\bar{z})^{2}}-16 \pi \frac{T(\bar{z})-2 p(\bar{z})}{\bar{z}^{3}(1-\bar{z})}\right.\left.\quad-\frac{4 \alpha}{\bar{z}}\right] d \bar{z}, \\
& h(z)=\int_{z}^{1}\left[\frac{2 \alpha\left(1-2 \bar{z}^{2}\right)}{\bar{z}^{2}(1-\bar{z})}-\frac{32 \pi \rho(\bar{z})}{\bar{z}^{4}}\right] d \bar{z} \\
&=\int_{z}^{1}\left[-32 \pi \frac{H(\bar{z})+G(\bar{z})}{\bar{z}^{2}(1-\bar{z})}+32 \pi \frac{T(\bar{z})-2 p(\bar{z})}{\bar{z}^{4}}\right. \\
&\left.\quad+4 \alpha \frac{(1+\bar{z})}{\bar{z}^{2}}\right] d \bar{z} .
\end{aligned}
$$

Note that the functions $H(z)$ and $G(z)$ are given in terms of $T(z)$ and $p(z)$ by Eqs. (39) and (41). For any conformally invariant quantum field, the trace of the stress tensor is known exactly and is given by the conformal anomaly. In the Schwarzschild spacetime, the dimensionless trace is $T(z)=\mu^{4} T_{\alpha}^{\alpha}$, where

$$
T(z)=\gamma z^{6}=\beta \xi z^{6},
$$


with $\beta \equiv 1 /\left(2^{13} 3^{2} 5 \pi^{2}\right)$. Here $\xi$ is a dimensionless coefficient of the trace. For spins $0,1 / 2,1,3 / 2$, and 2 (with particles identical to antiparticles, so that for each momentum, there is a single one-particle state for spin 0 and two for higher spins, one for each of the two helicities), $\xi$ is, respectively, 96, 168, $-1248,-5592$, and 20352 [49], but this does not give the full trace for gravitons, which are not conformally invariant [45]. Equation (39) then gives

$$
H(z)=\frac{\gamma}{10}\left(1-z^{5}\right)=\frac{\beta \xi}{10}\left(1-z^{5}\right)
$$

Therefore, we can derive $g(z)$ and $h(z)$ using these special forms for $T(z)$ and $H(z)$,

$$
\begin{array}{r}
g(z)=32 \pi \int_{z}^{1}\left(\frac{G(\bar{z})}{\bar{z}(1-\bar{z})^{2}}+\frac{p(\bar{z})}{\bar{z}^{3}(1-\bar{z})}\right) d \bar{z}+\frac{8}{15} \pi \gamma(1-z)\left(29+17 z+8 z^{2}\right)+\left(4 \alpha-\frac{16}{5} \pi \gamma\right) \ln z, \\
h(z)=32 \pi \int_{z}^{1} \frac{1}{\bar{z}^{4}}\left(2 p(\bar{z})+\frac{\bar{z}^{2}}{1-\bar{z}} G(\bar{z})\right) d \bar{z}+\frac{8}{5} \pi \gamma \frac{(1-z)}{z}\left(2-3 z-5 z^{2}-6 z^{3}\right)+\left(4 \alpha-\frac{16}{5} \pi \gamma\right) \ln z-\frac{4 \alpha}{z}(1-z) .
\end{array}
$$

A five-term polynomial is believed to be a good approximation for the function $p(z)$. There is evidence [63,64] that $p(z)$ may start off at order $z^{3}$ (but see below), and the anomalous trace introduces a term of $z^{6}$. Also, for spin-1 particles, there exists evidence [63] for a $z^{7}$ term. Therefore, we consider $p(z)$ to be a polynomial of the form

$$
p(z)=\beta\left(k_{3} z^{3}+k_{4} z^{4}+k_{5} z^{5}+k_{6} z^{6}+k_{7} z^{7}\right)
$$

Figure 2 gives graphs of $p(z) / z^{3}$ from numerical values of the $k_{i}$ coefficients given later, below Eq. (85). Using Eq. (74) for $p(z)$ in Eq. (41) gives

$$
\begin{aligned}
G(z)= & \beta(1-z)\left[\frac{k_{3}}{2}(1-3 z)-k_{4} z^{2}-\frac{k_{5}}{12}\left(1+z+z^{2}+9 z^{3}\right)\right. \\
& \left.-\frac{k_{6}}{10}\left(1+z+z^{2}+z^{3}+6 z^{4}\right)-\frac{k_{7}}{10}\left(1+z+z^{2}+z^{3}+z^{4}+5 z^{5}\right)\right] .
\end{aligned}
$$

Finally, we can write $f(z), \rho(z)$ and $P(z)$ in the following form:

$$
\begin{gathered}
f(z)=\beta f_{0} \frac{z^{2}}{1-z} \\
\rho(z)=-f(z)+\beta z^{2}\left[\frac{\xi}{10}\left(1+z+z^{2}+z^{3}-9 z^{4}\right)+\frac{k_{3}}{2}(1+z)+k_{4} z^{2}-\frac{k_{5}}{12}\left(1+z+z^{2}-15 z^{3}\right)\right. \\
\left.-\frac{k_{6}}{10}\left(1+z+z^{2}+z^{3}-14 z^{4}\right)-\frac{k_{7}}{10}\left(1+z+z^{2}+z^{3}+z^{4}-15 z^{5}\right)\right]
\end{gathered}
$$
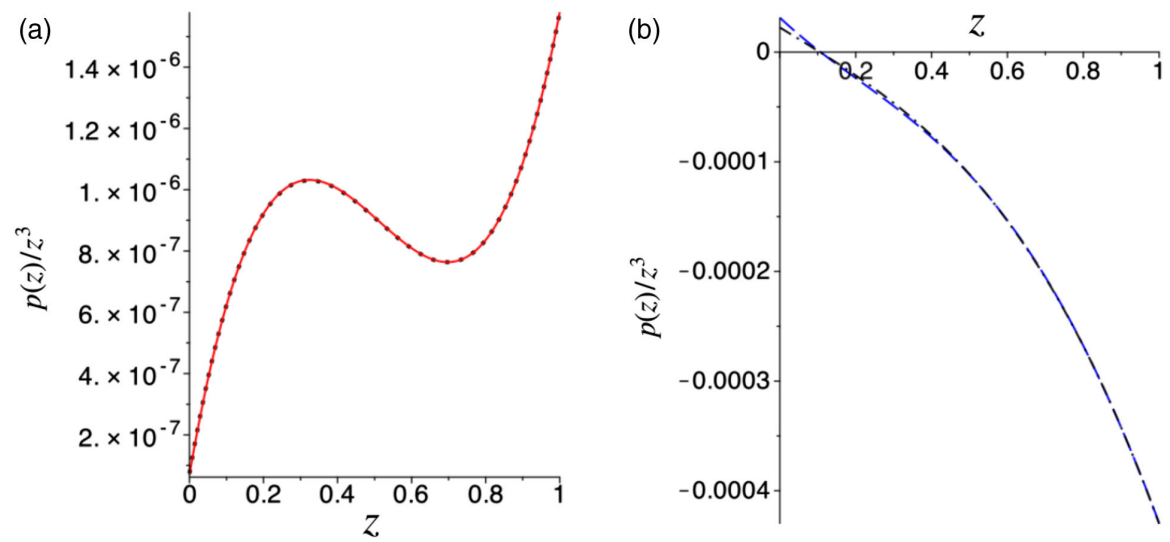

FIG. 2. (a) Behavior of the function $p(z) / z^{3}$ for spin 0 , black dotted line for the case of nonzero $k_{3}, k_{4}, k_{5}$, and $k_{6}$ given by [63] and red solid line for the case of nonzero $k_{3}, k_{4}, k_{5}$, and $k_{6}$ given by [64]. (b) Behavior of the function $p(z) / z^{3}$ for spin 1 , blue dashed line for the case of nonzero $k_{3}, k_{4}, k_{5}, k_{6}$, and $k_{7}$ given by [63] and black dash-dotted line for the case of nonzero $k_{3}, k_{4}, k_{5}$, and $k_{6}$ given by [64]. 


$$
\begin{aligned}
P(z)= & -f(z)+\beta z^{2}\left[\frac{\xi}{10}\left(1+z+z^{2}+z^{3}+z^{4}\right)+\frac{k_{3}}{2}(1-3 z)-k_{4} z^{2}-\frac{k_{5}}{12}\left(1+z+z^{2}+9 z^{3}\right)\right. \\
& \left.-\frac{k_{6}}{10}\left(1+z+z^{2}+z^{3}+6 z^{4}\right)-\frac{k_{7}}{10}\left(1+z+z^{2}+z^{3}+z^{4}+5 z^{5}\right)\right] .
\end{aligned}
$$

We want at asymptotic infinity the stress-energy tensor to be that of an outgoing flux of positive radiation, requiring $\rho(z) \rightarrow f(z)$ asymptotically as $z \rightarrow 0$ [3]. Picking up the dominant terms $\left[O\left(z^{2}\right)\right]$ in (76) and (77), we see that

$$
f_{0}=\frac{\xi}{20}+\frac{k_{3}}{4}-\frac{k_{5}}{24}-\frac{k_{6}}{20}-\frac{k_{7}}{20}
$$

In the case that $k_{3}=0$, this constraint is the same as Eq. (29) in [21]. If now we apply the constraint (79), we can replace one of the constants $\xi, k_{3}, k_{4}, k_{5}, k_{6}$, or $k_{7}$. For example, we can write

$$
\begin{aligned}
& \xi=20 f_{0}-5 k_{3}+\frac{5}{6} k_{5}+k_{6}+k_{7}=2^{11} 3^{2} 5^{2} \pi \alpha-5 k_{3}+\frac{5}{6} k_{5}+k_{6}+k_{7}, \\
& k_{6}=-20 f_{0}+\xi+5 k_{3}-\frac{5}{6} k_{5}-k_{7}=-2^{11} 3^{2} 5^{2} \pi \alpha+\xi+5 k_{3}-\frac{5}{6} k_{5}-k_{7}, \\
& k_{7}=-20 f_{0}+\xi+5 k_{3}-\frac{5}{6} k_{5}-k_{6}=-2^{11} 3^{2} 5^{2} \pi \alpha+\xi+5 k_{3}-\frac{5}{6} k_{5}-k_{6} .
\end{aligned}
$$

Then, the equations for $\rho(z)$ and $P(z)$ can be written as follows:

$$
\begin{aligned}
& \rho(z)=\frac{\beta z^{2}}{1-z}\left[\frac{\xi}{20}\left(1-20 z^{4}+18 z^{5}\right)+\frac{k_{3}}{4}\left(1-2 z^{2}\right)+k_{4} z^{2}(1-z)\right. \\
& \left.-\frac{k_{5}}{24}\left(1-32 z^{3}+30 z^{4}\right)-\frac{k_{6}}{20}\left(1-30 z^{4}+28 z^{5}\right)-\frac{k_{7}}{20}\left(1-32 z^{5}+30 z^{6}\right)\right] \\
& =\beta z^{2}\left[\frac{f_{0}}{1-z}\left(1-20 z^{4}+18 z^{5}\right)-\frac{k_{3}}{2} z^{2}\left(1+z-9 z^{2}\right)+k_{4} z^{2}+\frac{k_{5}}{12} z^{3}(16-9 z)+\frac{k_{6}}{2} z^{4}-\frac{k_{7}}{2} z^{4}(2-3 z)\right] \\
& =\beta z^{2}\left[\frac{f_{0}}{1-z}\left(1-30 z^{4}+28 z^{5}\right)+\frac{\xi}{2} z^{4}-\frac{k_{3}}{2} z^{2}\left(1+z-14 z^{2}\right)+k_{4} z^{2}+\frac{k_{5}}{6} z^{3}(8-7 z)-\frac{3 k_{7}}{2} z^{4}(1-z)\right] \\
& =\beta z^{2}\left[\frac{f_{0}}{1-z}\left(1-32 z^{5}+30 z^{6}\right)-\frac{\xi}{2} z^{4}(2-3 z)-\frac{k_{3}}{2} z^{2}\left(1+z+z^{2}-15 z^{3}\right)\right. \\
& \left.+k_{4} z^{2}+\frac{k_{5}}{12} z^{3}(1+z)(16-15 z)+\frac{3 k_{6}}{2} z^{4}(1-z)\right] \\
& P(z)=\frac{\beta z^{2}}{1-z}\left[\frac{\xi}{20}\left(1-2 z^{5}\right)+\frac{k_{3}}{4}\left(1-8 z+6 z^{2}\right)-k_{4} z^{2}(1-z)\right. \\
& \left.-\frac{k_{5}}{24}\left(1+16 z^{3}-18 z^{4}\right)-\frac{k_{6}}{20}\left(1+10 z^{4}-12 z^{5}\right)-\frac{k_{7}}{20}\left(1+8 z^{5}-10 z^{6}\right)\right] \\
& =\beta z^{2}\left[\frac{f_{0}}{1-z}\left(1-2 z^{5}\right)-\frac{k_{3}}{2} z\left(4+z+z^{2}+z^{3}\right)-k_{4} z^{2}-\frac{k_{5}}{12} z^{3}(8-z)-\frac{k_{6}}{2} z^{4}-\frac{k_{7}}{2} z^{5}\right] \\
& =\beta z^{2}\left[\frac{f_{0}}{1-z}\left(1+10 z^{4}-12 z^{5}\right)-\frac{\xi}{2} z^{4}-\frac{k_{3}}{2} z\left(4+z+z^{2}+6 z^{3}\right)-k_{4} z^{2}-\frac{k_{5}}{6} z^{3}(4-3 z)+\frac{k_{7}}{2} z^{4}(1-z)\right] \\
& =\beta z^{2}\left[\frac{f_{0}}{1-z}\left(1+8 z^{5}-10 z^{6}\right)-\frac{\xi}{2} z^{5}-\frac{k_{3}}{2} z\left(4+z+z^{2}+z^{3}+5 z^{4}\right)-k_{4} z^{2}-\frac{k_{5}}{12} z^{3}\left(8-z-5 z^{2}\right)-\frac{k_{6}}{2} z^{4}(1-z)\right] \text {. }
\end{aligned}
$$


Expressions (83) and (84) are each presented in four forms, first in terms of $\xi$ and the $k_{i}$ coefficients, then in terms of $f_{0}$ and the $k_{i}$ coefficients and then by substituting either $k_{6}$ or $k_{7}$ from the constraint. This is done because current fits to the numerical computations of the energy-momentum tensor show that for spin-0 particles an expansion of (74) with terms up to $k_{6}$ are appropriate. However, for spin-1 particles, an expansion of (74) with terms up to $k_{7}$ are used in [63]. Now, solving the Einstein equation (53), we obtain

$$
\begin{aligned}
g(z)= & \frac{1}{2^{11} 3^{3} 5^{2} \pi}(1-z)\left[2\left(\xi-k_{6}\right)\left(29+17 z+8 z^{2}\right)-5 k_{5}(7+3 z)-k_{7}\left(73+49 z+31 z^{2}+15 z^{3}\right)\right] \\
= & \frac{1}{2^{11} 3^{4} 5 \pi}(1-z)\left[6\left(4 f_{0}-k_{3}\right)\left(29+17 z+8 z^{2}\right)+8 k_{5}\left(1+z+z^{2}\right)-9 k_{7}(1+z)\left(1+z^{2}\right)\right] \\
= & \frac{1}{2^{12} 3^{4} 5 \pi}(1-z)\left[-18\left(\xi-k_{6}\right)(1+z)\left(1+z^{2}\right)+6\left(4 f_{0}-k_{3}\right)\left(73+49 z+31 z^{2}+15 z^{3}\right)\right. \\
& \left.+k_{5}\left(31+31 z+31 z^{2}+15 z^{3}\right)\right], \\
h(z)= & \frac{1}{2^{11} 3^{3} 5^{2} \pi}(1-z)\left[-6 \xi\left(3+5 z+6 z^{2}\right)+120 k_{4}+5 k_{5}(13+15 z)+2 k_{6}\left(19+25 z+28 z^{2}\right)\right. \\
& \left.+k_{7}\left(23+35 z+41 z^{2}+45 z^{3}\right)\right] \\
= & \frac{1}{2^{11} 3^{3} 5 \pi}(1-z)\left[-6\left(4 f_{0}-k_{3}\right)\left(3+5 z+6 z^{2}\right)+24 k_{4}+2 k_{5}\left(5+5 z-3 z^{2}\right)+4 k_{6}\left(1+z+z^{2}\right)\right. \\
& \left.+k_{7}\left(1+z+z^{2}+9 z^{3}\right)\right] \\
= & \frac{1}{2^{11} 3^{4} 5 \pi}(1-z)\left[12 \xi\left(1+z+z^{2}\right)-6\left(4 f_{0}-k_{3}\right)\left(19+25 z+28 z^{2}\right)\right. \\
& \left.+72 k_{4}+4 k_{5}\left(5+5 z-7 z^{2}\right)-9 k_{7}(1-z)\left(1+2 z+3 z^{2}\right)\right] \\
= & \frac{1}{2^{12} 3^{4} 5 \pi}(1-z)\left[6 \xi\left(1+z+z^{2}+9 z^{3}\right)-6\left(4 f_{0}-k_{3}\right)\left(23+35 z+41 z^{2}+45 z^{3}\right)\right. \\
& \left.+144 k_{4}+k_{5}\left(55+55 z-41 z^{2}-45 z^{3}\right)+18 k_{6}(1-z)\left(1+2 z+3 z^{2}\right)\right] .
\end{aligned}
$$

We remind the reader that $\xi$ is the value of the trace anomaly coefficient from Eq. (70). The constants $k_{3}, k_{4}, k_{5}$, $k_{6}$, and $k_{7}$ appear in Eq. (74) for the transverse pressure. The expressions for $g(z)$ and $h(z)$ are each presented in four forms, first in terms of $\xi$ and the $k_{i}$ coefficients, then in terms of $f_{0}$ and the $k_{i}$ coefficients, and then by substituting either $k_{6}$ or $k_{7}$ from the constraint (except that the second and third forms for $g(z)$ coincide, so that form is written only once). The constant $f_{0}$ is related to the Hawking luminosity by $L=-d m / d v \approx \alpha / \mu^{2}=16 \pi \beta f_{0} / \mu^{2}$, with $\beta \equiv 1 /\left(2^{13} 3^{2} 5 \pi^{2}\right)$.

For the case of a conformal scalar field (spin 0) in the Unruh state, Matt Visser [21] has performed a least-squares fit to the transverse pressure data of Jensen et al. [17] and of Anderson et al. [18], giving the constants $k_{4}=26.5652$, $k_{5}=-59.0214, k_{6}=38.2068$ (to six digits, though not claimed accurate beyond $1 \%$ ), and $f_{0}=5.349$ (close to Elster's value [8] of 5.385 that is probably more accurate). From the summary in [21] of the data of $[17,18]$ and of private communications to Visser from some of those authors, Bardeen discovered that an improved fit to that data and to the scalar luminosity results $[8,12,22]$ could be made by including a nonzero $k_{3}$, which he kindly provided us in 2016 [65] and published in 2017 [63]: $k_{3}=0.264$, $k_{4}=25.438, k_{5}=-57.460, k_{6}=37.503$, and $f_{0}=5.385$. In 2018, Bardeen [64] presented revised fits of $k_{3}=$ $0.2524, \quad k_{4}=25.5439, \quad k_{5}=-57.6663, \quad k_{6}=37.6172$, and $f_{0}=5.385$ for conformally invariant scalars (spin 0).

For the electromagnetic field (massless spin 1) in the Unruh state, Bardeen [63] used the results of Jensen and Ottewill [16] and Jensen et al. [17] to give $k_{3}=114.62$, $k_{4}=-1186.24, k_{5}=1393.96, k_{6}=-2537.42$ and $k_{7}=$ 652.20, with $f_{0}=2.435$. In 2018, Bardeen [64] obtained access provided privately by Visser from the calculations of [17], and this led to an improved fit with $k_{3}=81.80$, $k_{4}=-770.42, k_{5}=65.38$, and $k_{6}=-942.18$, with $f_{0}=$ 2.4346 (using data from [59]), with a nonzero $k_{7}$ no longer seen necessary. For our graphs, we use the Bardeen $[63,64]$ data as probably the most careful synthesis of previous calculations. Figures 3-5 graph $g(z), h(z)$, and $\mu^{2} \psi$ [given by Eq. (18)] for the numerical values of the $k_{i}$ coefficients.

Now that we have calculated the $h(z)$ and $g(z)$ functions of the metric (28), we can calculate the Kretschmann scalar $\mathcal{K} \equiv R_{\alpha \beta \gamma \delta} R^{\alpha \beta \gamma \delta}$, which has the following form: 


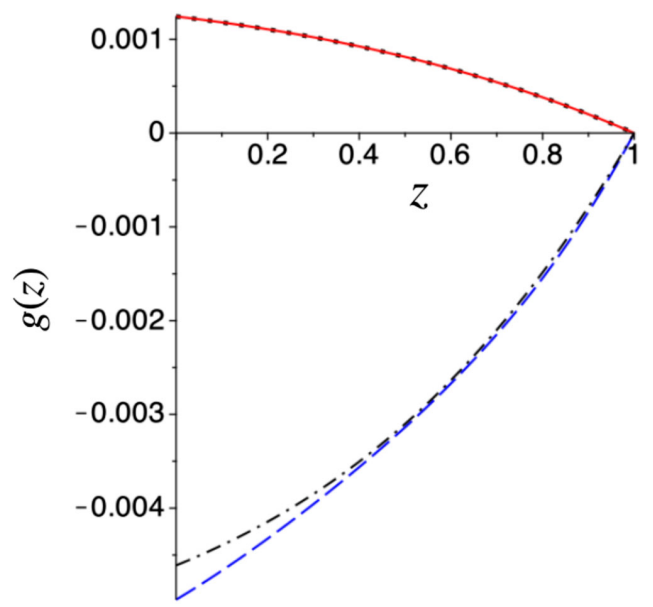

FIG. 3. Behavior of the function $g(z)$ for spin 0, black dotted line for the case of nonzero $k_{3}, k_{4}, k_{5}$, and $k_{6}$ given by [63], and red solid line for the case of nonzero $k_{3}, k_{4}, k_{5}$, and $k_{6}$ given by [64]. For spin 1, blue dashed line for the case of nonzero $k_{3}, k_{4}$, $k_{5}, k_{6}$, and $k_{7}$ given by [63] and black dash-dotted line for the case of nonzero $k_{3}, k_{4}, k_{5}$, and $k_{6}$ given by [64].

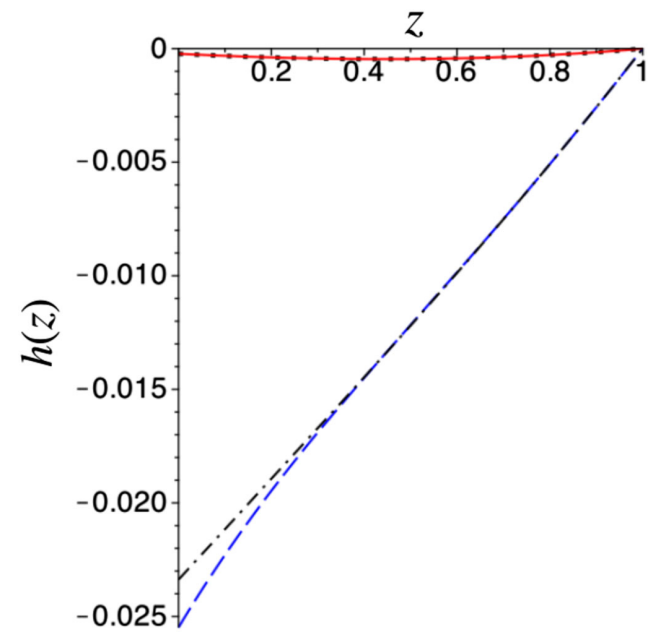

FIG. 4. Behavior of the function $h(z)$ for spin 0, black dotted line for the case of nonzero $k_{3}, k_{4}, k_{5}$, and $k_{6}$ given by [63] and red solid line for the case of nonzero $k_{3}, k_{4}, k_{5}$, and $k_{6}$ given by [64]. For spin 1, blue dashed line for the case of nonzero $k_{3}, k_{4}$, $k_{5}, k_{6}$, and $k_{7}$ given by [63] and black dash-dotted line for the case of nonzero $k_{3}, k_{4}, k_{5}$, and $k_{6}$ given by [64].

$$
\begin{aligned}
\mathcal{K} & =12 F^{2}+4 F\left(2 G_{a}^{a}-G_{i}^{i}\right)+\left(G_{a}^{a}-G_{i}^{i}\right)^{2}+2 G_{b}^{a} G_{a}^{b} \\
& =12 F^{2}+8 F\left(G_{0}^{0}+G_{1}^{1}-G_{2}^{2}\right)+3\left(G_{0}^{0}\right)^{2}+3\left(G_{1}^{1}\right)^{2}+2 G_{0}^{0} G_{1}^{1}+4 G_{1}^{0} G_{0}^{1}-4 G_{2}^{2}\left(G_{0}^{0}+G_{1}^{1}-G_{2}^{2}\right) \\
& =12 F^{2}+8 F\left(G_{r}^{r}+G_{v}^{v}-G_{\theta}^{\theta}\right)+3\left(G_{r}^{r}\right)^{2}+3\left(G_{v}^{v}\right)^{2}+2 G_{r}^{r} G_{v}^{v}+4 G_{v}^{r} G_{r}^{v}-4 G_{\theta}^{\theta}\left(G_{r}^{r}+G_{v}^{v}-G_{\theta}^{\theta}\right) \\
& \approx \frac{48 m^{2}}{r^{6}}-\frac{128 \pi m}{r^{3} \mu_{0}^{4}}[p(z)-P(z)+\rho(z)] .
\end{aligned}
$$

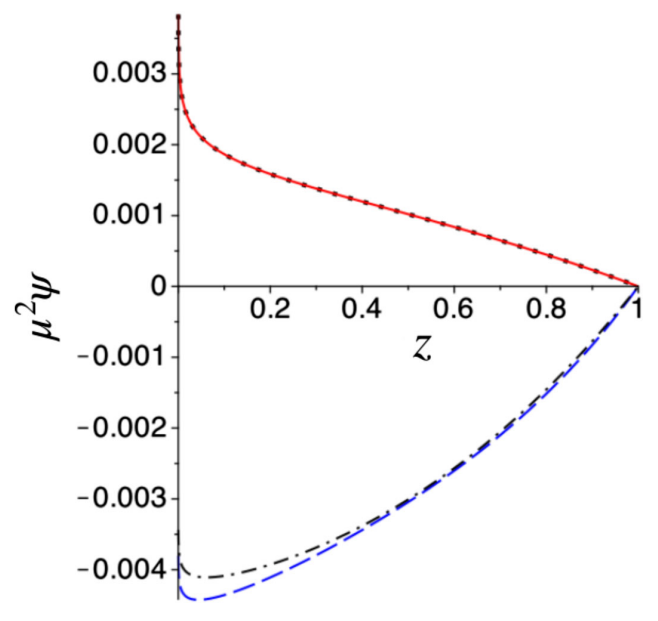

FIG. 5. Behavior of the function $\mu^{2} \psi$ for spin 0 , black dotted line for the case of nonzero $k_{3}, k_{4}, k_{5}$, and $k_{6}$ given by [63] and red solid line for the case of nonzero $k_{3}, k_{4}, k_{5}$, and $k_{6}$ given by [64]. For spin 1, blue dashed line for the case of nonzero $k_{3}, k_{4}$, $k_{5}, k_{6}$, and $k_{7}$ given by [63] and black dash-dotted line for the case of nonzero $k_{3}, k_{4}, k_{5}$, and $k_{6}$ given by [64].
Here, $F=2 m / r^{3}$, and the indices $a$ and $b$ correspond to 0 and 1 while $i$ and $j$ correspond to 2 and 3 . The first term in $\mathcal{K}$ is equal to the Kretschmann scalar for the Schwarzschild solution. In Fig. 6, we have plotted

$$
\begin{aligned}
C & \equiv r^{6} \mathcal{K}-48 m^{2} \approx \frac{2^{10} \pi}{z^{3}}[P(z)-\rho(z)-p(z)] \\
& =\frac{2^{10} \pi}{z^{3}}[T(z)-3 p(z)] .
\end{aligned}
$$

We further define

$$
S(z)=\mu_{0}^{8}\left(T^{a b}-\frac{1}{2} T_{c}^{c} g^{a b}\right)\left(T_{a b}-\frac{1}{2} T_{d}^{d} g_{a b}\right),
$$

where lowercase Latin letters $a, b$, etc., run only over 0 and 1 , or $t$ and $r$. In terms of

$$
\begin{aligned}
J(z)= & \frac{\rho+P+2 f}{\beta z^{2}(1-z)}=4 f_{0}\left(1+2 z+3 z^{2}+4 z^{3}\right) \\
& -k_{3}\left(2 z+3 z^{2}+4 z^{3}\right)+\frac{2}{3} k_{5} z^{3}-k_{7} z^{4},
\end{aligned}
$$

this gives 

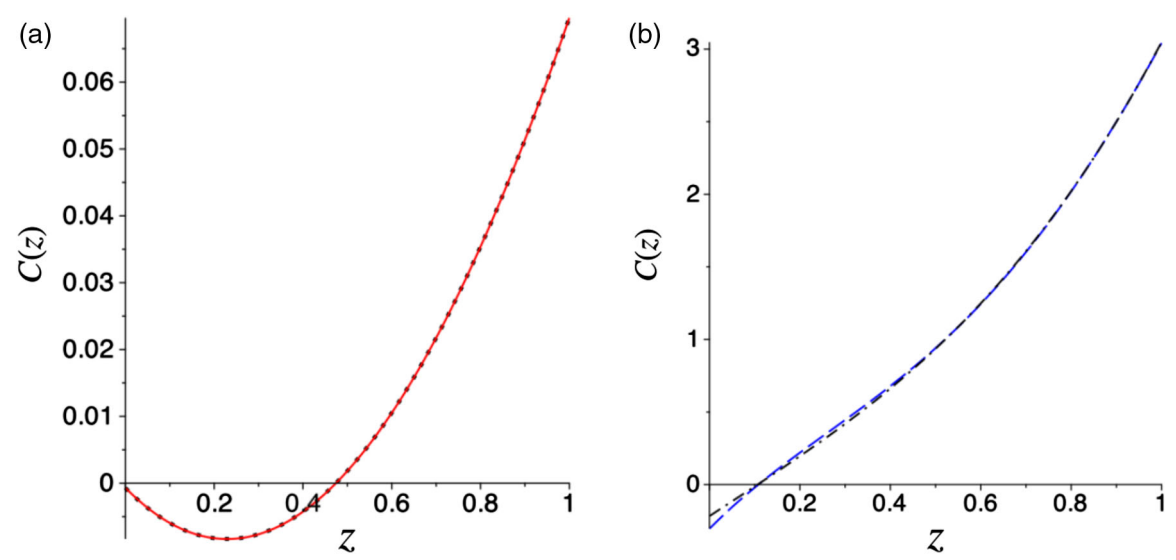

FIG. 6. (a) Behavior of the function $C \equiv r^{6} R_{\alpha \beta \gamma \delta} R^{\alpha \beta \gamma \delta}-48 m^{2} \approx 2^{10} \pi z^{-3}[P(z)-\rho(z)-p(z)]$ for spin 0 , black dotted line for the case of nonzero $k_{3}, k_{4}, k_{5}$, and $k_{6}$ given by [63] and red solid line for the case of nonzero $k_{3}, k_{4}, k_{5}$, and $k_{6}$ given by [64]. (b) Behavior of the function $C$ for spin 1, blue dashed line for the case of nonzero $k_{3}, k_{4}, k_{5}, k_{6}$, and $k_{7}$ given by [63] and black dash-dotted line for the case of nonzero $k_{3}, k_{4}, k_{5}$, and $k_{6}$ given by [64].

$$
\begin{aligned}
S(z)= & \frac{1}{2}(\rho+P)^{2}-2 f^{2}=\frac{1}{2} \beta^{2} z^{4} J(z)\left[(1-z)^{2} J(z)-4 f_{0}\right] \\
= & \frac{1}{18} \beta^{2} z^{5}\left[12 f_{0}\left(1+2 z+3 z^{2}+4 z^{3}\right)-3 k_{3}\left(2 z+3 z^{2}+4 z^{3}\right)+2 k_{5} z^{3}-3 k_{7} z^{4}\right] \\
& \times\left[-12 f_{0}\left(5 z^{3}-4 z^{4}\right)-3 k_{3}\left(2-z-5 z^{3}+4 z^{4}\right)+2 k_{5}\left(z^{2}-2 z^{3}+z^{4}\right)-3 k_{7}\left(z^{3}-2 z^{4}+z^{5}\right)\right] \\
= & \beta^{2}\left[-4 f_{0} k_{3} z^{5}+\left(-6 f_{0} k_{3}+2 k_{3}^{2}\right) z^{6}+\left(-8 f_{0} k_{3}+\frac{4}{3} f_{0} k_{5}+2 k_{3}^{2}\right) z^{7}+\left(-40 f_{0}^{2}-2 f_{0} k_{7}+\frac{5}{2} k_{3}^{2}-\frac{4}{3} k_{3} k_{5}\right) z^{8}\right. \\
& +\left(-48 f_{0}^{2}+40 f_{0} k_{3}-7 k_{3}^{2}+\frac{2}{3} k_{3} k_{5}+2 k_{3} k_{7}\right) z^{9}+\left(-56 f_{0}^{2}+28 f_{0} k_{3}-\frac{7}{2} k_{3}^{2}-k_{3} k_{7}+\frac{2}{9} k_{5}^{2}\right) z^{10} \\
& +\left(-64 f_{0}^{2}+32 f_{0} k_{3}-\frac{40}{3} f_{0} k_{5}-4 k_{3}^{2}+\frac{10}{3} k_{3} k_{5}-\frac{4}{9} k_{5}^{2}-\frac{2}{3} k_{5} k_{7}\right) z^{11} \\
& +\left(128 f_{0}^{2}-64 f_{0} k_{3}+\frac{32}{3} f_{0} k_{5}+20 f_{0} k_{7}+8 k_{3}^{2}-\frac{8}{3} k_{3} k_{5}-5 k_{3} k_{7}+\frac{2}{9} k_{5}^{2}+\frac{4}{3} k_{5} k_{7}+\frac{1}{2} k_{7}^{2}\right) z^{12} \\
& \left.+\left(-16 f_{0} k_{7}+4 k_{3} k_{7}-\frac{2}{3} k_{5} k_{7}-k_{7}^{2}\right) z^{13}+\frac{1}{2} k_{7}^{2} z^{14}\right] .
\end{aligned}
$$

Near the horizon, for $z \rightarrow 1$, we have

$$
S(z) \sim \beta^{2} f_{0}\left[-80 f_{0}+18 k_{3}-\frac{4}{3} k_{5}+2 k_{7}+\left(480 f_{0}-112 k_{3}+\frac{28}{3} k_{5}-16 k_{7}\right)(1-z)\right] .
$$

Figure 7 shows the behavior of $J(z)$, and Fig. 8 graphs $S(z) / z^{5}$. Note also that very far from the black hole, $z \ll 1$, the leading contributions (lowest powers of $z$ ) are $S(z) \sim$ $-4 \beta^{2} f_{0} k_{3} z^{5}+\beta^{2}\left(-6 f_{0} k_{3}+2 k_{3}^{2}\right) z^{6}+\beta^{2}\left(-8 k_{3} f_{0}+(4 / 3) \times\right.$ $\left.f_{0} k_{5}+2 k_{3}^{2}\right) z^{7}$. Since for both spin 0 and spin 1 , Bardeen's fits give $k_{3}>0$, if there is indeed such a positive $1 / r^{3}$ term in the transverse stress, this would imply that $S(z)$ is negative for sufficiently small $z$ (large $r /\left(2 \mu_{0}\right) \equiv 1 / z$ ). However, the $k_{3} z^{3}$ term in Bardeen's fits to the transverse stress does not dominate over the $k_{4} z^{4}$ term for either the conformal massless spin 0 or the massless spin 1 until one goes to values of $r$ much larger than the range where the stress could be calculated numerically. Therefore, although this term indeed improves the fit for the finite range of $r$ where the numerical calculations were made, possible small systematic errors in these calculations might have been responsible for the fit by a nonzero $k_{3} z^{3}$ term, rather than indicating an actual asymptotic term of this form [66]. Marolf [67] and Horowitz [68] have expressed scepticism about the existence of a $1 / r^{3}$ term in the transverse stress, and now Ori [69] has given us a detailed argument against such a term. Therefore, if indeed asymptotically $k_{3}=0$, the leading term for $S(z)$ would be $(4 / 3) \beta^{2} f_{0} k_{5} z^{7}$, which is negative for conformal massless spin 0 but positive for massless spin 1 . 

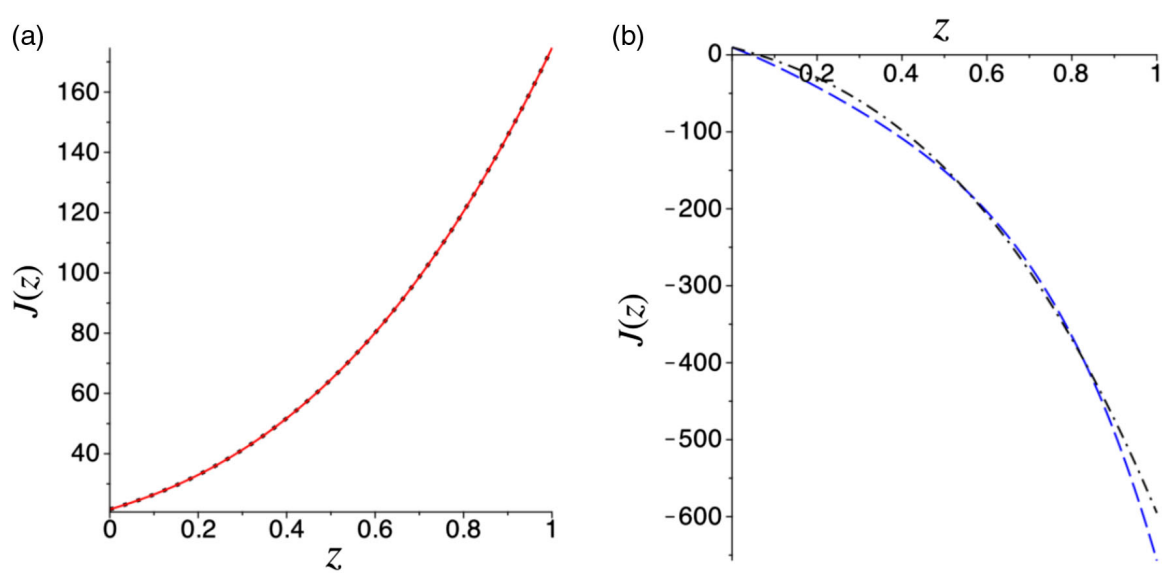

FIG. 7. (a) Behavior of the function $J(z)$ for spin 0 , black dotted line for the case of nonzero $k_{3}, k_{4}, k_{5}$, and $k_{6}$ given by [63] and red solid line for the case of nonzero $k_{3}, k_{4}, k_{5}$, and $k_{6}$ given by [64]. (b) Behavior of the function $J(z)$ for spin 1 , blue dashed line for the case of nonzero $k_{3}, k_{4}, k_{5}, k_{6}$, and $k_{7}$ given by [63] and black dash-dotted line for the case of nonzero $k_{3}, k_{4}, k_{5}$, and $k_{6}$ given by [64].
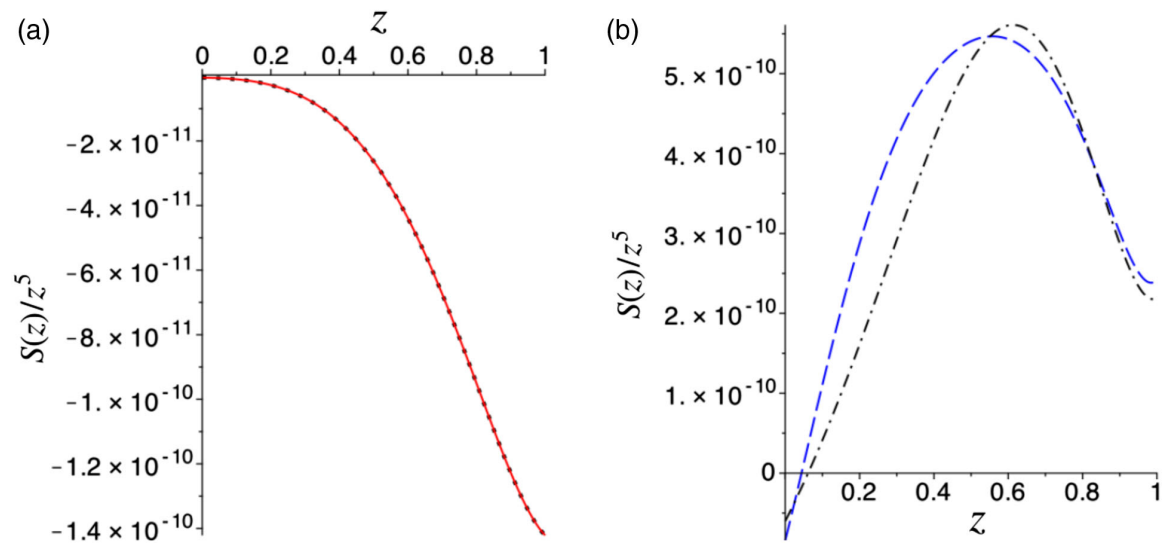

FIG. 8. (a) Behavior of the function $S(z) / z^{5}$ for spin 0 , black dotted line for the case of nonzero $k_{3}, k_{4}, k_{5}$, and $k_{6}$ given by [63] and red solid line for the case of nonzero $k_{3}, k_{4}, k_{5}$, and $k_{6}$ given by [64]. For spin $0, S(z)<0$ for $0<z<1$, implying that the stress-energy tensor is of Hawking-Ellis Type IV [70] with no timelike or null eigenvector, so there is no frame without energy flux anywhere outside the black hole for the massless conformal scalar field for both cases of Bardeen's fitting coefficients and also for the earlier fit by Visser [21] with $k_{3}=0$. (b) Behavior of the function $S(z) / z^{5}$ for spin 1 , blue dashed line for the case of nonzero $k_{3}, k_{4}, k_{5}, k_{6}$, and $k_{7}$ given by [63] and black dash-dotted line for the case of nonzero $k_{3}, k_{4}, k_{5}$, and $k_{6}$ given by [64]. For $p(z)$ given by Eq. (74) for spin 1 with nonzero $k_{3}, k_{4}, k_{5}, k_{6}$, and $k_{7}$ given by [63], $S(z)>0$ for $z>0.04374$, but $S(z)<0$ for $0<z<0.04374$, where there is no frame with zero energy flux. For the spin-1 approximation for $p(z)$ with nonzero $k_{3}, k_{4}, k_{5}$, and $k_{6}$ given by [64], $S(z)>0$ for $z>0.06151$, but $S(z)<0$ for $0<z<0.06151$. However, if actually $k_{3}=0$, it is not yet clear whether or not there is any region with $S(z)<0$ for spin 1 (the electromagnetic field) that would give a Hawking-Ellis Type IV [70] stress-energy tensor with no timelike eigenvector.

Negative $S(z)$ means that the stress-energy tensor is of Hawking-Ellis Type IV [70], so there is no timelike or null eigenvector. This implies that no matter how fast an observer moves away from the black hole where $S(z)<0$, the Hawking radiation energy flux in the observer's frame will always be outward (unlike solar radiation, e.g., where at an average location of the earth at one astronomical unit from the sun, and using the value of the solar radius from [71], an observer with an outward velocity greater than $0.9999937453(12)$ of the speed of light [a gamma factor greater than $\gamma=283.737(26) \approx 3^{1 / 4}$ (astronomical unit)/ (solar radius)] relative to the sun, will see the sun cover enough of sky [more than $63.4 \%$ ] that the flux will be inward in the observer's frame). Therefore, if indeed there were a nonzero $k_{3} z^{3}$ term asymptotically for the transverse stress of the positive sign as given by Bardeen's fits [63,64], then for both the massless conformal spin 0 and spin 1 , the stressenergy tensor would be asymptotically Type IV, with no frame in which the flux is zero, but if $k_{3}=0$, then the stress-energy tensor will be asymptotically Type IV for the conformal spin 0 with $k_{5}<0$ but the ordinary Type I (with a timelike eigenvector, for which an observer whose 4-velocity is the timelike eigenvector of the stress-energy tensor would see zero flux) asymptotically for massless spin 1 with $k_{5}>0$. 
Numerically, we find that the stress-energy tensor for the massless conformal scalar is Type IV everywhere outside the horizon for both of Bardeen's fits [63,64] and also for the earlier fit by Visser [21] with $k_{3}=0$. However, for massless spin 1, it is only Type IV for $0<z<0.04374$ for [63] or for $0<z<0.06151$ for [64], and since it would not be Type IV for sufficiently small $z$ if $k_{3}=0$ (assuming $k_{5}>0$ as given by both $[63,64])$, we are not certain that the stress-energy tensor for the electromagnetic field in the Unruh state is Type IV (no timelike eigenvector) anywhere outside a slowly evaporating Schwarzschild black hole.

Here we should note that we disagree with the results [35], who claim that the stress-energy tensor for a conformally coupled massless scalar field is Type I (the usual type, with a timelike eigenvector) near the horizon, for $z \gtrsim 0.9843$. Their $\Gamma$, defined by their
Eq. (2.28) to be the same as twice our $S$, is given by a semianalytical model in their Eq. (7.41) (which seems to have many incorrect coefficients), and apparently these mistakes and/or round-off errors lead to the nonzero coefficient 0.9434 for the unphysical divergence at the horizon given by their Eq. (7.42). We believe that we are the first to show that a conformally coupled massless scalar field in the Unruh state has a stress-energy tensor that is Hawking-Ellis Type IV [70] everywhere outside the horizon of a slowly evaporating Schwarzschild black hole, so that there are no observers anywhere outside that see zero energy flux.

Another scalar that we can calculate now that we know the form of the $h(z)$ and $g(z)$ functions is $\mathcal{T}^{2} \equiv \mu_{0}^{8} T^{\mu \nu} T_{\mu \nu}$, which goes into the trace-of-square energy condition (TOSEC) of [35], that $\mathcal{T}^{2} \geq 0$,

$$
\begin{aligned}
& \mathcal{T}^{2}(z)=\rho^{2}+P^{2}+2 p^{2}-2 f^{2}=S+2 p^{2}+\frac{1}{2}(2 p-T)^{2} . \\
& \mathcal{T}^{2}(z)= \beta^{2}\left[-4 f_{0} k_{3} z^{5}+\left(-6 f_{0} k_{3}+6 k_{3}^{2}\right) z^{6}+\left(-8 f_{0} k_{3}+\frac{4}{3} f_{0} k_{5}+2 k_{3}^{2}+8 k_{3} k_{4}\right) z^{7}\right. \\
&+\left(-40 f_{0}^{2}-2 f_{0} k_{7}+\frac{5}{2} k_{3}^{2}+\frac{20}{3} k_{3} k_{5}+4 k_{4}^{2}\right) z^{8} \\
&+\left(-48 f_{0}^{2}+3 k_{3}^{2}-k_{3} k_{5}+6 k_{3} k_{6}+8 k_{4} k_{5}\right) z^{9} \\
&+\left(-56 f_{0}^{2}+28 f_{0} k_{3}-40 f_{0} k_{4}-\frac{7}{2} k_{3}^{2}+10 k_{3} k_{4}+7 k_{3} k_{7}-\frac{5}{3} k_{4} k_{5}+6 k_{4} k_{6}-2 k_{4} k_{7}+\frac{38}{9} k_{5}^{2}\right) z^{10} \\
&+\left(-64 f_{0}^{2}+32 f_{0} k_{3}-\frac{160}{3} f_{0} k_{5}-4 k_{3}^{2}+\frac{40}{3} k_{3} k_{5}+8 k_{4} k_{7}-\frac{19}{9} k_{5}^{2}+6 k_{5} k_{6}-\frac{8}{3} k_{5} k_{7}\right) z^{11} \\
&+\left(328 f_{0}^{2}-164 f_{0} k_{3}+\frac{82}{3} f_{0} k_{5}-20 f_{0} k_{6}+40 f_{0} k_{7}\right. \\
&\left.+\frac{41}{2} k_{3}^{2}-\frac{41}{6} k_{3} k_{5}+5 k_{3} k_{6}-10 k_{3} k_{7}+\frac{41}{72} k_{5}^{2}-\frac{5}{6} k_{5} k_{6}+\frac{61}{6} k_{5} k_{7}+\frac{5}{2} k_{6}^{2}-k_{6} k_{7}+k_{7}^{2}\right) z^{12} \\
&\left.+\left(-56 f_{0} k_{7}+14 k_{3} k_{7}-\frac{7}{3} k_{5} k_{7}+6 k_{6} k_{7}-3 k_{7}^{2}\right) z^{13}+\frac{9}{2} k_{7}^{2} z^{14}\right] .
\end{aligned}
$$

Near the horizon, for $z \rightarrow 1$, we have

$$
\begin{aligned}
\mathcal{T}^{2}(z) & \sim \beta^{2}\left(120 f_{0}^{2}-122 f_{0} k_{3}-40 f_{0} k_{4}-\frac{74}{3} f_{0} k_{5}-20 f_{0} k_{6}-18 f_{0} k_{7}+\frac{53}{2} k_{3}^{2}+18 k_{3} k_{4}+\frac{73}{6} k_{3} k_{5}+11 k_{3} k_{6}\right. \\
& \left.+11 k_{3} k_{7}+4 k_{4}^{2}+\frac{19}{3} k_{4} k_{5}+6 k_{4} k_{6}+6 k_{4} k_{7}+\frac{193}{72} k_{5}^{2}+\frac{31}{6} k_{5} k_{6}+\frac{31}{6} k_{5} k_{7}+\frac{5}{2} k_{6}^{2}+5 k_{6} k_{7}+\frac{5}{2} k_{7}^{2}\right) \\
& -\beta^{2}\left(1920 f_{0}^{2}-1448 f_{0} k_{3}-400 f_{0} k_{4}-\frac{748}{3} f_{0} k_{5}-240 f_{0} k_{6}-264 f_{0} k_{7}+264 k_{3}^{2}\right. \\
& +156 k_{3} k_{4}+109 k_{3} k_{5}+114 k_{3} k_{6}+132 k_{3} k_{7}+32 k_{4}^{2}+\frac{166}{3} k_{4} k_{5}+60 k_{4} k_{6}+68 k_{4} k_{7} \\
& \left.+\frac{155}{6} k_{5}^{2}+56 k_{5} k_{6}+\frac{187}{3} k_{5} k_{7}+30 k_{6}^{2}+66 k_{6} k_{7}+36 k_{7}^{2}\right)(1-z) .
\end{aligned}
$$

Figure 9 shows the behavior of $\mathcal{T}^{2}(z) / z^{5}$. 

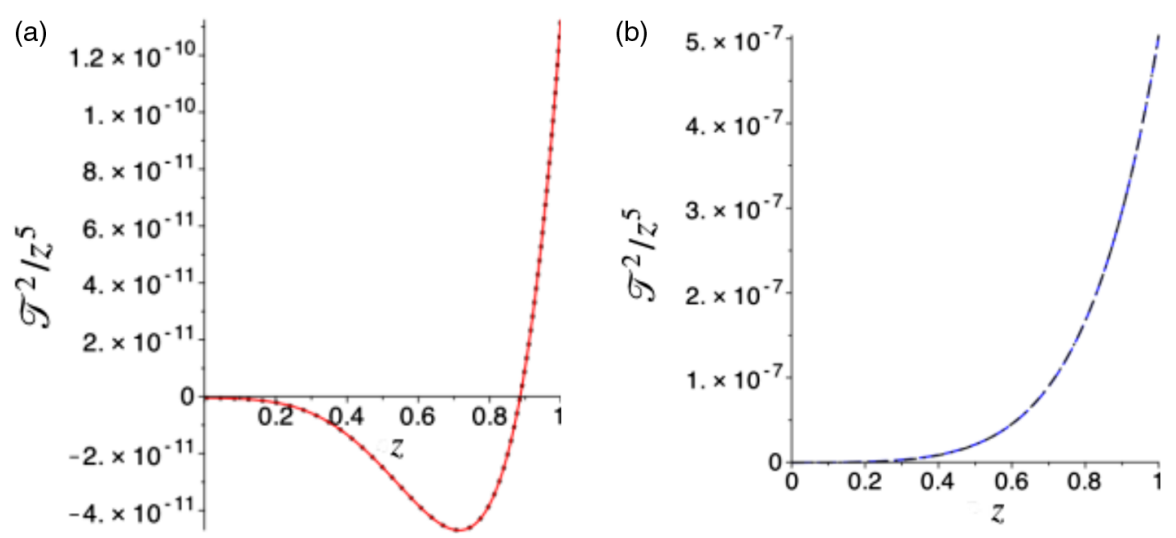

FIG. 9. (a) Behavior of the function $\mathcal{T}^{2}(z) / z^{5}$ for spin 0 , black dotted line for the case of nonzero $k_{3}, k_{4}, k_{5}$, and $k_{6}$ given by [63] and red solid line for the case of nonzero $k_{3}, k_{4}, k_{5}$, and $k_{6}$ given by [64]. For $p(z)$ given by Eq. (74) for spin 0 with nonzero $k_{3}, k_{4}, k_{5}, k_{6}$, and $k_{7}$ given by [63], $\mathcal{T}^{2}(z)>0$ for $z>0.88587$, but $\mathcal{T}^{2}(z)<0$ for $0<z<0.88587$, violating the TOSEC of [35]. For the spin-0 approximation for $p(z)$ with nonzero $k_{3}, k_{4}, k_{5}$, and $k_{6}$ given by [64], $\mathcal{T}^{2}(z)>0$ for $z>0.88585$, but $\mathcal{T}^{2}(z)<0$ for $0<z<0.88585$. (b) Behavior of the function $\mathcal{T}^{2}(z) / z^{5}$ for spin 1, blue dashed line for the case of nonzero $k_{3}, k_{4}, k_{5}, k_{6}$, and $k_{7}$ given by [63] and black dash-dotted line for the case of nonzero $k_{3}, k_{4}, k_{5}$, and $k_{6}$ given by [64]. For $p(z)$ given by Eq. (74) for spin 1 with nonzero $k_{3}, k_{4}, k_{5}, k_{6}$, and $k_{7}$ given by [63], $\mathcal{T}^{2}(z)>0$ for $z>0.01873$, but $\mathcal{T}^{2}(z)<0$ for $0<z<0.01873$. For the spin-1 approximation for $p(z)$ with nonzero $k_{3}, k_{4}, k_{5}$, and $k_{6}$ given by [64], $\mathcal{T}^{2}(z)>0$ for $z>0.03039$, but $\mathcal{T}^{2}(z)<0$ for $0<z<0.03039$. However, if actually $k_{3}=0$, it is not yet clear whether or not there is any region with $\mathcal{T}^{2}(z)<0$ for spin 1 (the electromagnetic field) that would violate the TOSEC of $[35]$.

Numerically, we have that for the conformal massless scalar field, $\mathcal{T}^{2}(z)<0$ (violating the TOSEC defined by [35]) for $z<0.88587$ and $\mathcal{T}^{2}(z)>0$ (the more common situation, obeying the TOSEC) for $0.88587<z<1$ from the fitting data of [63], or $\mathcal{T}^{2}(z)<0$ for $z<0.88585$ and $\mathcal{T}^{2}(z)>0$ for $0.88585<z<1$ from the fitting data of [64]. For the massless vector field, $\mathcal{T}^{2}(z)<0$ for $z<$ 0.01873 and $\mathcal{T}^{2}(z)>0$ for $0.01873<z<1$ from the fitting data of [63], or $\mathcal{T}^{2}(z)<0$ for $z<0.03039$ and $\mathcal{T}^{2}(z)>0$ for $0.03039<z<1$ from the fitting data of [64]. However, these results assume the positive values of $k_{3}$ from Bardeen's fits $[63,64]$, so that although with the more robust result that $k_{5}<0$ for the conformal massless scalar, the TOSEC for it seems to be violated at large distances from the black hole even with $k_{3}=0$, for the massless vector field (e.g., the electromagnetic field) with $k_{5}>0$, it is less clear whether or not the TOSEC is violated anywhere outside an evaporating black hole.

A lacuna in our numerical results is that there is as yet, so far as we know, no good approximations for the effective stress-energy tensor components from the spin-2 quantum gravitational field (other than for the flux coefficient $f_{0}$ ). Assuming that photons and gravitons are the only massless fields in nature and that the lightest neutrino mass is not orders of magnitude smaller than the others so that it would be comparable to or less than the Hawking temperature of an astrophysical black hole, once we know the spin-2 stress-energy tensor of the Unruh state around a Schwarzschild black hole, then after the Universe expands sufficiently, and after matter clears out from near each astrophysical black hole, then our formulas would enable one to calculate an accurate metric (i.e., one whose small departure at each time from the Schwarzschild metric is accurately known) for such an isolated astrophysical black hole as it evaporates by the emission of Hawking radiation that would be almost entirely photons and gravitons.

So far, we have found the metric for $1 \ll(-3 \alpha v)^{\frac{1}{3}} \lesssim$ $r \ll-3 v / 2$. (Indeed, it should apply even a bit inside the event horizon that is very near $z=1$, but for $z$ significantly larger than 1, the approximations used for the stress-energy tensor will not be valid.) However, we have assumed that $2 r \ll-3 v$ so that the difference between $\mu(v, z)$ and $\mu_{0}(v) \equiv(-3 \alpha v)^{\frac{1}{3}}$ is small, and so that $\tilde{z}$ in Eq. (18) is approximately $z \equiv 2 \mu / r \approx 2 \mu_{0} / r$. Now it is time to find what $\tilde{z}$ is when these approximations are not valid, in order that we may have expressions for the metric coefficients that are good approximations no matter how large $r$ is for fixed $v$ and hence no matter how small $z$ is. [Note that if $-4 \alpha \ln \tilde{z}$ were replaced by $-4 \alpha \ln z$ in Eq. (18), then $\psi$ would diverge as $r$ is taken to infinity and $z$ to zero, whereas actually $\psi$ stays finite and small.]

In a coordinate basis using the ingoing EddingtonFinkelstein coordinates $(v, r, \theta, \phi)$ of the metric (17), the Einstein equation gives

$$
\psi_{, r}=4 \pi r T_{r r}
$$

Alternatively, in an orthonormal frame in which $e_{\hat{0}}$ is the 4-velocity of worldlines of constant $(r, \theta, \phi)$ and $e_{\hat{1}}$ is the unit spacelike vector in the outward radial direction orthogonal to $e_{\hat{0}}$, one has 


$$
\psi_{, r}=\frac{4 \pi r}{1-2 m / r}\left(T^{\hat{0} \hat{0}}+T^{\hat{0} \hat{1}}+T^{\hat{1} \hat{0}}+T^{\hat{1} \hat{1}}\right) .
$$

For the polynomial approximations for the stress-energy tensor given above, one gets that the radial partial derivative of $\psi$ at fixed ingoing null coordinate $v, \psi_{, r}$, is approximately $1 /\left(\mu^{2} r\right)$ multiplied by a polynomial in $z=2 \mu / r$. Note that we have made the gauge choice of setting $\psi(v, z=1)=0$ at the apparent horizon $(z \equiv 2 \mu / r=1)$, so that the value of $\psi(v, z)$ outside the apparent horizon (i.e., for $z<1$ ) is obtained by integrating $\psi_{, r} d r$ from the apparent horizon at $r=2 \mu$ to the greater value $r=2 \mu / z$ along a null geodesic with constant $v$. Because the integrand is approximately $1 /\left(\mu^{2} r\right)$ multiplied by a polynomial in $z$, we can integrate $1 /\left(\mu^{2} r\right)$ multiplied by each power of $z$ and then combine the results with the appropriate coefficients from the polynomial in $z$. When we do this for the positive powers of $z, \mu$ will stay very near $\mu_{0} \equiv(-3 \alpha v)^{\frac{1}{3}} \gg 1$ for nearly all of the dominant part of the integral, so that those integrals will combine to give $g(z) / \mu_{0}^{2}$. However, if we assume that $\mu \approx \mu_{0}$ when $1 /\left(\mu^{2} r\right)$ is multiplied by the constant term in the polynomial in $z$, this term integrates to $\left(-4 \alpha / \mu_{0}^{2}\right) \ln z$, which diverges as $r$ is taken to infinity and hence $z$ is taken to zero. This divergent logarithmic integral is of course dominated by very large $r$, where it is not valid to retain the approximation $\mu \approx \mu_{0}$, which is actually only valid for $2 r \ll-3 v$. For larger $r, \mu$ rises with $r$, so that the integral of $d r /\left(\mu^{2} r\right)$ remains bounded by a finite quantity no matter how large the upper limit of $r$ is taken. What we actually get is the convergent integral

$$
\left(-4 \alpha / \mu_{0}^{2}\right) \ln \tilde{z}=\int_{2 \mu_{0}}^{r} \frac{4 \alpha d r^{\prime}}{\mu^{2} r^{\prime}} .
$$

This integral will give $\tilde{z} \approx z=2 \mu / r$ for $2 r \ll-3 v$. The deviations will only become significant for larger $r$, for which the $1 / z$ term on the right-hand side of Eq. (5) becomes large in comparison with each of the other two terms. Then we can let

$$
x \equiv \frac{\mu}{\mu_{0}} \approx\left(1+\frac{6 \alpha r}{\mu_{0}{ }^{3}}\right)^{\frac{1}{3}}=\left(1+\frac{2 r}{-v}\right)^{\frac{1}{3}}
$$

become the new independent variable, which has a lower limit at $r=2 \mu_{0}$ of $x_{h}=\left(1+12 \alpha / \mu_{0}^{2}\right)^{\frac{1}{3}} \approx 1+4 \alpha / \mu_{0}^{2}$, which then gives

$$
-\ln \tilde{z}=\int_{x_{h}}^{x} \frac{3 d y}{y^{3}-1}
$$

and hence $\tilde{z} \approx \frac{4 \alpha}{\mu_{0}^{2}(x-1)} \sqrt{\frac{x^{2}+x+1}{3}} \exp \left[\sqrt{3} \tan ^{-1}\left(\frac{1}{\sqrt{3}} \frac{x-1}{x+1}\right)\right]$.

For $6 \alpha r \ll \mu_{0}^{3} \equiv-3 \alpha v$, so that $x \approx 1+2 \alpha r / \mu_{0}^{3}$ is very near 1 , this gives $\tilde{z} \approx z$, but unlike $z$, which goes to zero as $r$ goes to infinity, $\tilde{z}$ decreases only to the very small positive $v$-dependent constant

$$
\begin{aligned}
\tilde{z}_{0}(v) & \equiv \tilde{z}(v, z=0) \approx \frac{4 \alpha}{\sqrt{3} \mu_{0}^{2}} \exp \left(\frac{\sqrt{3} \pi}{6}\right) \\
& =\frac{4 \alpha^{1 / 3}}{3^{7 / 6}(-v)^{2 / 3}} \exp \left(\frac{\sqrt{3} \pi}{6}\right) .
\end{aligned}
$$

As a result, the function $\psi$ in the ingoing EddingtonFinkelstein metric (17), which we have set to zero [along with $g(z)$ and $h(z)$ ] at the apparent horizon at $z=1$, does not diverge as $r$ is taken to infinity and $z \equiv 2 \mu / r$ is taken to zero, but rather it goes to the finite (and small) limit of

$$
\psi_{0}(v) \equiv \psi(v, z=0) \approx \frac{\left(g_{0}-4 \alpha \ln \tilde{z_{0}}\right)}{\mu_{0}^{2}} .
$$

In Figs. (3-5), we have plotted the functions $g(z), h(z)$, and $\mu^{2} \psi$ for spin- 0 and spin- 1 particles, assuming that $\mu_{0}$ is sufficiently large that the last plot does not extend into the very large $r \gtrsim-v$ regime where $\mu$ significantly exceeds $\mu_{0}$.

Therefore, knowing the value of the trace anomaly coefficients $\xi$ for the appropriate conformal massless fields, i.e., scalar and/or electromagnetic, and the constants $k_{3}, k_{4}, k_{5}, k_{6}$, and $k_{7}$, we have an approximate timedependent metric for an evaporating black hole as a firstorder perturbation of the Schwarzschild metric given by Eqs. (17)-(19) in $(v, r)$ coordinates with $z \equiv 2 \mu / r$ from Eq. (6), $\mu(v, z)$ from Eqs. (7)-(9), or $\mu(v, r)$ from Eq. (11) (approximately for $r \ll \mu^{3} / \alpha$ ), the functions $g(z)$ and $h(z)$ given by Eq. (85), and the function $\tilde{z}$ given by Eq. (100) with $x$ therein given by Eq. (98). We also remind the reader that we have chosen the gauge such that at the apparent horizon, where $z=1$ in our approximate metric, $\epsilon(1)=$ $g(1)=h(1)=\ln \tilde{z}(1)=0$, and that this metric applies everywhere outside the black hole before it gets so small that quantum gravity effects become important.

\section{COMPARISON OF METRICS}

The metric (17) with our expressions for the functions contained therein should be valid for $1 \ll(-3 \alpha v)^{\frac{1}{3}} \lesssim r$. (Indeed, it should apply also for somewhat smaller $r$, a bit inside the event horizon that is very near $z=1$, but for $z$ significantly larger than 1 , the approximations used for the stress-energy tensor will not be valid.) Next, we want to convert it back to outgoing Eddington-Finkelstein coordinates to see how our metric behaves in the coordinate 
system $(u, r)$ in the restricted region $\mu / r=(-3 \alpha u)^{1 / 3} /$ $r \ll 1$, where the outgoing Vaidya metric (1) is approximately valid. Consider the metric (17) with functions $h$ and $g$ known from Eq. (85), using the relation

$$
v \approx u+2 r-4(-3 \alpha u)^{1 / 3}+4(-3 \alpha u)^{1 / 3} \ln \left[\frac{r}{2(-3 \alpha u)^{1 / 3}}\right] .
$$

We get

$$
d v=\Psi_{1} d u+\Psi_{2} d r
$$

where

$$
\begin{gathered}
\Psi_{1} \approx 1-\frac{4 \alpha \ln \left[\frac{r}{2(-3 \alpha u)^{1 / 3}}\right]}{(-3 \alpha u)^{2 / 3}}+\frac{8 \alpha}{(-3 \alpha u)^{2 / 3}}, \\
\Psi_{2} \approx 2+\frac{4(-3 \alpha u)^{1 / 3}}{r} .
\end{gathered}
$$

The metric (17) with functions $h$ and $g$ given by Eq. (85) becomes

$$
\begin{aligned}
d s^{2}= & -e^{2 \psi}\left(1-\frac{2 m}{r}\right) \Psi_{1}^{2} d u^{2} \\
& -2\left[e^{2 \psi}\left(1-\frac{2 m}{r}\right) \Psi_{1} \Psi_{2}-e^{\psi} \Psi_{1}\right] d u d r \\
& +\left[2 e^{\psi} \Psi_{2}-e^{2 \psi}\left(1-\frac{2 m}{r}\right) \Psi_{2}^{2}\right] d r^{2}+r^{2} d \Omega^{2} \\
= & g_{u u} d u^{2}+2 g_{u r} d u d r+g_{r r} d r^{2}+r^{2} d \Omega^{2} .
\end{aligned}
$$

For $-3 \alpha u \gg 1$, we have

$$
\begin{gathered}
g_{u u}=-1+\frac{2(-3 \alpha u)^{1 / 3}}{r}+\frac{1}{(-3 \alpha u)^{1 / 3} r}[32 \alpha+2 h+4 g] \\
+\mathcal{O}\left(\frac{1}{(-3 \alpha u)^{2 / 3}}\right) \\
g_{u r}=-1+\frac{8(-3 \alpha u)^{2 / 3}}{r^{2}}+\mathcal{O}\left(\frac{1}{r^{2}}\right) \\
g_{r r}=\frac{16(-3 \alpha u)^{2 / 3}}{r^{2}}+\frac{-96 \alpha u}{r^{3}}+\mathcal{O}\left(\frac{(-3 \alpha u)^{4 / 3}}{r^{4}}\right)
\end{gathered}
$$

Equations (107)-(110) give us the corrections to the outgoing Vaidya metric (1) for an evaporating black hole in outgoing Eddington-Finkelstein coordinates. When we consider only terms of the order of unity and of first order in the small quantity $\mu / r=(-3 \alpha u)^{1 / 3} / r$, we get

$$
\begin{gathered}
g_{u u} \approx-1+\frac{2(-3 \alpha u)^{1 / 3}}{r}, \\
g_{u r} \approx-1, \\
g_{r r} \approx 0 .
\end{gathered}
$$

Therefore, for $\mu / r=(-3 \alpha u)^{1 / 3} / r \ll 1$, we get the outgoing Vaidya metric,

$d s^{2} \approx\left(-1+\frac{2(-3 \alpha u)^{1 / 3}}{r}\right) d u^{2}-2 d u d r+r^{2} d \Omega^{2}$.

This form of the metric applies for $1 \ll(-3 \alpha v)^{\frac{1}{3}} \ll r$, so it does not apply near the black hole horizon, where the more general Eq. (17) does apply, but it is applicable for arbitrarily large $r$. It also applies for positive advanced time $v$, after the black hole has evaporated, so long as one avoids the Planckian region near the final evaporation and its causal future, where quantum gravity effects are expected.

\section{SUMMARY}

In this paper, we have constructed an approximate timedependent metric for an evaporating black hole as a firstorder perturbation of the Schwarzschild metric, using the linearized backreaction from the stress-energy tensor of the Hawking radiation in the Unruh quantum state in the unperturbed spacetime. We used a metric ansatz in ingoing Eddington-Finkelstein coordinates $(v, r)$. Our ansatz is such that at infinity we get the Vaidya metric in the outgoing Eddington-Finkelstein coordinates $(u, r)$. We have solved the corresponding Einstein equations for the metric functions to first order in the stress-energy tensor of the unperturbed Schwarzschild metric. Therefore, our metric should be a very good approximation everywhere near to and everywhere outside the event horizon when the mass is large in Planck units.

\section{ACKNOWLEDGMENTS}

We acknowledge helpful information about the stressenergy tensor by email from James Bardeen. This work was supported in part by the Natural Sciences and Engineering Research Council of Canada. During the latter stages of the paper, D. N.P. appreciated the hospitality of the Perimeter Institute and of Matthew Kleban at the Center for Cosmology and Particle Physics of New York University. Research at Perimeter Institute is supported by the Government of Canada through the Department of Innovation, Science and Economic Development and by the Province of Ontario through the Ministry of Research, Innovation and Science. Calculations by D. N. P. during one stage of the paper were made at the Cook's Branch Nature Conservancy under the generous hospitality of the Mitchell family and of the George P. and Cynthia W. 
Mitchell Institute for Fundamental Physics and Astronomy of Texas A \& M University. Private communications of S. A. with Donald Marolf were during S. A.'s visit to the Kavli Institute for Theoretical Physics (KITP) at the
University of California, Santa Barbara. S. A. used the KITP's facilities as a KITP scholar supported in part by the National Science Foundation under Grant No. NSF PHY-1748958.
[1] S. W. Hawking, Particle creation by black holes, Commun. Math. Phys. 43, 199 (1975).

[2] S. M. Christensen, Vacuum expectation value of the stress tensor in an arbitrary curved background: The covariant point separation method, Phys. Rev. D 14, 2490 (1976).

[3] S. M. Christensen and S. A. Fulling, Trace anomalies and the Hawking effect, Phys. Rev. D 15, 2088 (1977).

[4] W. A. Hiscock, Models of evaporating black holes, Phys. Rev. D 23, 2813 (1981).

[5] W. A. Hiscock, Models of evaporating black holes. II. Effects of the outgoing created radiation, Phys. Rev. D 23, 2823 (1981).

[6] D. N. Page, Thermal stress tensors in static Einstein spaces, Phys. Rev. D 25, 1499 (1982).

[7] V. P. Frolov and A. I. Zelnikov, Vacuum polarization by a massive scalar field in Schwarzschild space-time, Phys. Lett. B 115, 372 (1982).

[8] T. Elster, Vacuum polarization near a black hole creating particles, Phys. Lett. 94A, 205 (1983).

[9] K. W. Howard and P. Candelas, Quantum Stress Tensor in Schwarzschild Space-Time, Phys. Rev. Lett. 53, 403 (1984).

[10] T. Zannias, Renormalized thermal stress tensor for arbitrary static space-times, Phys. Rev. D 30, 1161 (1984).

[11] K. W. Howard, Vacuum $\left\langle T_{\mu}{ }^{\nu}\right\rangle$ in Schwarzschild space-time, Phys. Rev. D 30, 2532 (1984).

[12] R. D. Simkins, Massive scalar particle emission from Schwarzschild black holes, Ph.D. thesis, Pennsylvania State University, University Park, Pennsylvania, 1986.

[13] M. R. Brown, A. C. Ottewill, and D. N. Page, Conformally invariant quantum field theory in static Einstein space-times, Phys. Rev. D 33, 2840 (1986).

[14] V. P. Frolov and A. I. Zelnikov, Quantum energy momentum tensor in space-time with timelike Killing vector, Phys. Lett. B 193, 171 (1987).

[15] V. P. Frolov and A. I. Zelnikov, Killing approximation for vacuum and thermal stress-energy tensor in static spacetimes, Phys. Rev. D 35, 3031 (1987).

[16] B. Jensen and A. Ottewill, Renormalized electromagnetic stress tensor in Schwarzschild space-time, Phys. Rev. D 39, 1130 (1989).

[17] B. P. Jensen, J. G. McLaughlin, and A. C. Ottewill, Renormalized electromagnetic stress tensor for an evaporating black hole, Phys. Rev. D 43, 4142 (1991).

[18] P. R. Anderson, W. A. Hiscock, and D. A. Samuel, Stress Energy Tensor of Quantized Scalar Fields in Static Black Hole Space-Times, Phys. Rev. Lett. 70, 1739 (1993).

[19] P. R. Anderson, W. A. Hiscock, and D. A. Samuel, Stress-energy tensor of quantized scalar fields in static spherically symmetric space-times, Phys. Rev. D 51, 4337 (1995).

[20] J. Matyjasek, Stress energy tensor of an electromagnetic field in Schwarzschild space-time, Phys. Rev. D 55, 809 (1997).

[21] M. Visser, Gravitational vacuum polarization. 4: Energy conditions in the Unruh vacuum, Phys. Rev. D 56, 936 (1997).

[22] B. E. Taylor, C. M. Chambers, and W. A. Hiscock, Evaporation of a Kerr black hole by emission of scalar and higher spin particles, Phys. Rev. D 58, 044012 (1998).

[23] J. Matyjasek, $\left\langle T_{\mu \nu}\right\rangle_{\text {ren }}$ of the quantized conformal fields in the Unruh state in the Schwarzschild space-time, Phys. Rev. D 59, 044002 (1999).

[24] P. B. Groves, P. R. Anderson, and E. D. Carlson, Method to compute the stress-energy tensor for the massless spin $1 / 2$ field in a general static spherically symmetric spacetime, Phys. Rev. D 66, 124017 (2002).

[25] E. D. Carlson, W. H. Hirsch, B. Obermayer, P. R. Anderson, and P. B. Groves, Stress Energy Tensor for the Massless Spin 1/2 Field in Static Black Hole Spacetimes, Phys. Rev. Lett. 91, 051301 (2003).

[26] J. Matyjasek, Approximate stress-energy tensor of the massless spin-1/2 field in Schwarzschild spacetime, Phys. Rev. D 71, 027504 (2005).

[27] A. A. Popov, Analytical approximation of the stress energy tensor of a quantized scalar field in static spherically symmetric space-times, Phys. Rev. D 67, 044021 (2003).

[28] M. Casals and A. C. Ottewill, Canonical quantization of the electromagnetic field on the Kerr background, Phys. Rev. D 71, 124016 (2005).

[29] D. Morgan, S. Thom, E. Winstanley, and P. M. Young, Some general properties of the renormalized stress-energy tensor for static quantum states on $(n+1)$-dimensional spherically symmetric black holes, Gen. Relativ. Gravit. 39, 1719 (2007).

[30] C. Breen and A. C. Ottewill, Extended Green-Liouville asymptotics and vacuum polarization for lukewarm black holes, Phys. Rev. D 82, 084019 (2010).

[31] A. C. Ottewill and P. Taylor, Vacuum polarization on the Schwarzschild metric threaded by a cosmic string, Phys. Rev. D 82, 104013 (2010).

[32] A. C. Ottewill and P. Taylor, Renormalized vacuum polarization and stress tensor on the horizon of a Schwarzschild black hole threaded by a cosmic string, Classical Quantum Gravity 28, 015007 (2011).

[33] C. Breen and A. C. Ottewill, Hadamard renormalisation of the stress energy tensor on the horizons of a spherically 
symmetric black hole space-time, Phys. Rev. D 85, 064026 (2012).

[34] C. Breen and A. C. Ottewill, Hadamard renormalization of the stress energy tensor in a spherically symmetric black hole space-time with an application to lukewarm black holes, Phys. Rev. D 85, 084029 (2012).

[35] P. Martin-Moruno and M. Visser, Semiclassical energy conditions for quantum vacuum states, J. High Energy Phys. 09 (2013) 050.

[36] A. Belokogne and A. Folacci, Renormalized stress tensor for massive fields in Kerr-Newman spacetime, Phys. Rev. D 90, 044045 (2014).

[37] A. Levi and A. Ori, Pragmatic mode-sum regularization method for semiclassical black-hole spacetimes, Phys. Rev. D 91, 104028 (2015).

[38] H. R. C. Ferreira, Quantum field theory on rotating black hole spacetimes, arXiv:1509.07683.

[39] A. Levi and A. Ori, Mode-sum regularization of $\left\langle\phi^{2}\right\rangle$ in the angular-splitting method, Phys. Rev. D 94, 044054 (2016).

[40] J. Matyjasek, Stress-energy tensor of the quantized massive scalar field in spherically symmetric, topological and lukewarm black hole configurations in $D=4$ and $D=5$, Phys. Rev. D 94, 084048 (2016).

[41] A. Levi and A. Ori, Versatile Method for Renormalized Stress-Energy Computation in Black-Hole Spacetimes, Phys. Rev. Lett. 117, 231101 (2016).

[42] A. Levi, E. Eilon, A. Ori, and M. van de Meent, Renormalized Stress-Energy Tensor of an Evaporating Spinning Black Hole, Phys. Rev. Lett. 118, 141102 (2017).

[43] A. Levi, Renormalized stress-energy tensor for stationary black holes, Phys. Rev. D 95, 025007 (2017).

[44] D. M. Capper and M. J. Duff, Trace anomalies in dimensional regularization, Nuovo Cimento A 23, 173 (1974).

[45] R. Critchley, Trace anomaly for gravitons, Phys. Rev. D 18, 1849 (1978).

[46] J. B. Hartle and S. W. Hawking, Path-integral derivation of black-hole radiance, Phys. Rev. D 13, 2188 (1976).

[47] W. G. Unruh, Notes on black-hole evaporation, Phys. Rev. D 14, 870 (1976).

[48] W. G. Unruh, Origin of the particles in black hole evaporation, Phys. Rev. D 15, 365 (1977).

[49] N. D. Birrell and P.C.W. Davies, Quantum Fields in Curved Space (Cambridge University Press, Cambridge, United Kingdom, 1982).

[50] J. W. York, Jr., Black hole in thermal equilibrium with a scalar field: The back reaction, Phys. Rev. D 31, 775 (1985).

[51] P. R. Anderson, W. A. Hiscock, J. Whitesell, and J. W. York, Jr., Semiclassical black hole in thermal equilibrium with a nonconformal scalar field, Phys. Rev. D 50, 6427 (1994).

[52] D. Hochberg and S. V. Sushkov, Black hole in thermal equilibrium with a spin-2 quantum field, Phys. Rev. D 53, 7094 (1996).

[53] D. Hochberg and T. W. Kephart, Gauge field back reaction on a black hole, Phys. Rev. D 47, 1465 (1993).

[54] D. Hochberg, T. W. Kephart, and J. W. York, Jr., Positivity of entropy in the semiclassical theory of black holes and radiation, Phys. Rev. D 48, 479 (1993).

[55] D. Hochberg, T. W. Kephart, and J. W. York, Jr., Effective potential of a black hole in thermal equilibrium with quantum fields, Phys. Rev. D 49, 5257 (1994).

[56] W. A. Hiscock, S. L. Larson, and P. R. Anderson, Semiclassical effects in black hole interiors, Phys. Rev. D 56, 3571 (1997).

[57] J. M. Bardeen, Black Holes Do Evaporate Thermally, Phys. Rev. Lett. 46, 382 (1981).

[58] D. N. Page, Particle emission rates from a black hole: Massless particles from an uncharged, nonrotating hole, Phys. Rev. D 13, 198 (1976).

[59] D. N. Page, Particle emission rates from a black hole. 2. Massless particles from a rotating hole, Phys. Rev. D 14, 3260 (1976).

[60] D. N. Page, Particle emission rates from a black hole. 3. Charged leptons from a nonrotating hole, Phys. Rev. D 16, 2402 (1977).

[61] D. N. Page, Comment on 'Entropy Evaporated by a Black Hole,' Phys. Rev. Lett. 50, 1013 (1983).

[62] D. N. Page, Hawking radiation and black hole thermodynamics, New J. Phys. 7, 203 (2005).

[63] J. M. Bardeen, The semi-classical stress-energy tensor in a Schwarzschild background, the information paradox, and the fate of an evaporating black hole, arXiv:1706.09204.

[64] J. M. Bardeen, Interpreting the semi-classical stress-energy tensor in a Schwarzschild background, implications for the information paradox, arXiv:1808.08638.

[65] J. M. Bardeen (private communication).

[66] J. M. Bardeen (private communication).

[67] D. Marolf (private communication).

[68] G. Horowitz (private communication).

[69] A. Ori (private communication).

[70] S. W. Hawking and G. F. R. Ellis, The Large Scale Structure of Space-Time (Cambridge University Press, Cambridge, United Kingdom, 1973), p. 90.

[71] M. Emilio, J. R. Kuhn, R. I. Bush, and I. F. Scholl, Measuring the solar radius from space during the 2003 and 2006 Mercury transits, Astrophys. J. 750, 135 (2012). 